\title{
Tools on the surface: residue and use-wear analyses of stone artefacts from Camooweal, northwest
} Queensland

\author{
Jane L. Cooper and Suzanne J. Nugent \\ School of Social Science \\ University of Queensland \\ St Lucia QLD 4072 Australia \\ Email: s.nugent@uq.edu.au
}

\begin{abstract}
Although much of the Australian archaeological record lies on the surface, such assemblages are often seen as having reduced archaeological potential when compared with subsurface deposits. However, a microscopic residue and use-wear analysis of surface-collected Aboriginal stone tulas from Camooweal, Queensland, revealed use-related residues including blood, bone collagen, woody plant tissue and resin, along with use-wear indicative of adzing and scraping functions. The results suggest the tulas were employed primarily for butchery, bone-working and woodworking tasks and were hafted in various orientations to the handle. Residues and use-wear were also detected on blades, hand axes, points, and cores from the same site, demonstrating that a large range of residues survive on artefacts from both surface and subsurface sites, enabling the reconstruction of details of subsistence and tool use.
\end{abstract}

\section{KEYWORDS}

tulas, use-wear, residue analysis, surface collected artefacts, hafting, woodworking, boneworking

\section{INTRODUCTION}

The techniques of residue and use-wear analysis have commonly been applied to stone artefacts from excavated contexts, where organic components and wear patterns relating to their use are considered more likely to have been preserved (Loy and Nugent 2002:20). Taphonomic processes acting upon surface artefacts have been considered too substantial to warrant this type of analysis (Davis 1975:52; Barton this volume; however see Briuer 1976). Despite advances in understanding post-depositional disturbance processes (see Ebert 1992; Fanning and Holdaway 2001; Greenfield 2000; Rossignol and Wandsnider 1992; Sullivan 1998; Wilkinson 2001), excavated materials continue to provide the major source of evidence for inferring past lifeways, and this bias is particularly evident on the scale of the individual artefact. This study draws on documented observations of tula use as an integral component of the Aboriginal toolkits to explore the legitimacy of applying microscopic residue and use-wear analyses to surface collected examples of these stone artefacts. We are greatly indebted to Dr Tom Loy for instigating the analysis of artefacts in the field and inspiring us to continue with his research. 


\section{SITE BACKGROUND}

The project area is situated along the construction corridor of a new $425 \mathrm{~m}$ two-lane bridge crossing the upper Georgina River at Camooweal, approximately $13 \mathrm{~km}$ from the Queensland / Northern Territory border (Figure 1). The Georgina River, banked mainly by box-eucalypt woodland, riparian woodland and Mitchell grasslands, consists of a chain of water holes during the dry winter months that are joined by floods during the wet season from January to February. Decomposition of the predominantly dolomite terrain has produced extensive residual deposits of nodular and tabular chert, known locally as 'ribbonstone', that is mantled by a thick, heavy clay soil. Many of the stone artefacts found in extensive surface scatters across the project area were knapped from this chert (Archaeo Cultural Heritage Services and Dugalunji Aboriginal Corporation 2002; Orr and Holmes 1990:243).

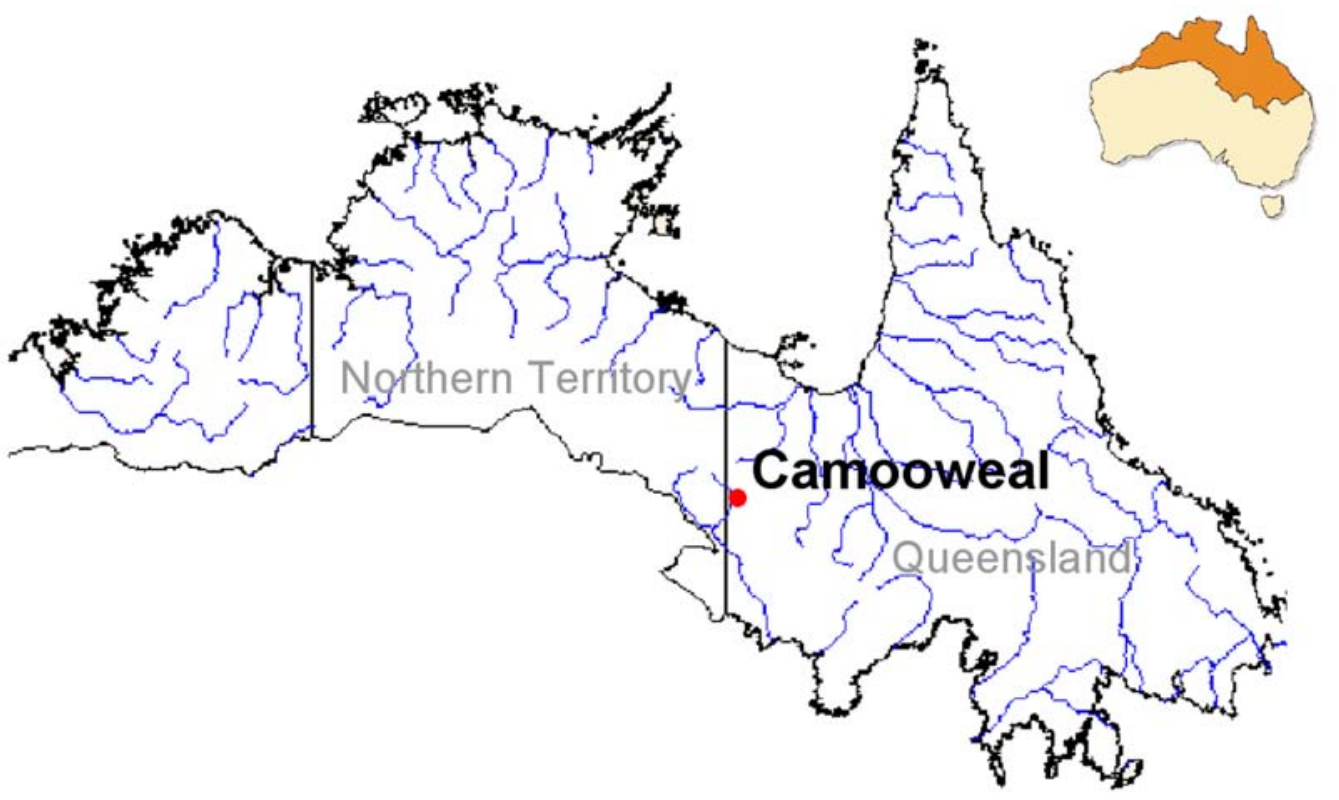

Figure 1. Map of northern Australia showing the location of Camooweal (after Tropical Savannas Cooperative Research Centre 2008).

Artefacts found on the surface were collected from a grid of $25 \mathrm{~m}$ x $50 \mathrm{~m}$ cells across the 3.825 ha site. In addition, 381 metre square pits were excavated, eight of which were randomly selected on the bridge pier footprints. Excavations usually terminated at $30 \mathrm{~cm}$. The entire area of the footprints was subsequently excavated with shovels and bobcats to the same depth and the removed soil sieved to recover artefacts. In general, a mixture of Aboriginal stone artefacts and modern and historical materials were distributed vertically, although not evenly, through the excavated soil to similar depths. A total of 16,645 stone artefacts was recovered from the area, among which were 8 hand-axes, 7,789 retouched flakes, 6,765 blades, and 917 tulas (Archaeo Cultural Heritage Services and Dugalunji Aboriginal Corporation 2002:31-3,37,42).

When it rains, the predominantly black soil of the Georgina River area rapidly becomes saturated. The high clay content impedes percolation and run-off with water tending to pool on the surface. When the saturated soil swells, buried stones are forced upwards towards the surface. During the dry season, the moisture evaporates, resulting in soil shrinkage and cracking. Some of the cracks observed during fieldwork were $4 \mathrm{~cm}$ wide at the surface and extend as deep as $40 \mathrm{~cm}$. These cracks provide an explanation for the migration of artefacts down the soil column. In addition, some mixing of artefacts would have occurred on the surface and approximately the top $10 \mathrm{~cm}$ of soil during grading of the road reserve (Archaeo Cultural Heritage Services and Dugalunji Aboriginal Corporation 2002:37-8). This post-depositional disturbance process has 
resulted in a site that is clearly disturbed and complex, as many excavated artefacts are likely to have previously seen multiple surface exposures, while those labelled 'surface collected' are just as likely to have been buried at some stage.

\section{PRELIMINARY ANALYSIS}

A total of 23 stone artefacts were examined first in the field (at Camooweal) and subsequently at the Archaeological Science Laboratory, University of Queensland in order to assess the potential for residue and use-wear analyses (Loy and Nugent 2002). The sample comprised four cores, 11 blades, four hand-axes and four tulas from surface and excavated contexts. The results are presented in Tables 1 and 2. In the field, an Olympus BHS microscope (50x, 100x, 200x, 500x and $800 x$ nominal magnifications) was used to examine at least one surface of 10 of these tools. For the remainder of this sample, a Wild stereo-binocular low-magnification microscope (6-30.6x) was used to observe indications of use-wear and traces of residues. An Olympus BX60 microscope $(50 x, 100 x, 200 x, 500 x, 1000 x)$ with incident light was used to identify residues and observe very fine striations on those artefacts other than the hand-axe (3666-5). Photographs were taken at all stages of the analysis using microscope-mounted Olympus DP10 cameras. Slides were prepared of residues extracted from artefact surfaces, mounted with Aquamount (refractive index $=1.400$ ), and examined using a BX60 microscope with transmitted light.

All 23 artefacts display possible use-wear marks and residues indicative of use. More importantly, when the results of the surface collected artefacts were compared to the excavated tools, some interesting trends became apparent. All four cores display indications of woodworking, and one surface and one excavated core also have evidence indicative of bone-working. The blades appear to have been used predominantly for bone- and woodworking, although two of the surface blades retain blood residues. It was inferred that residues including diatoms (naviculoid, cymbelloid and elongate types), sponge spicules, algae and plant tissue that were observed (in addition to bone collagen) on excavated blade 22666-2 are likely to result from regular inundation of the site by the Georgina River rather than use of the artefact for aquatic plant processing. Starch and plant tissue were identified on one of the surface hand-axes, suggesting the same task association as the two excavated hand axes, and all four tulas appear to have been employed in woodworking. Such consistent indications of use found on both the surface and excavated artefacts stimulated a detailed analysis of a further 16 surface collected tulas from the Camooweal site (Tables 1 and 2).

\section{TULA ANALYSIS}

\section{Ethnographic background}

The tula is one of the most common and distinctive chipped stone tools represented ethnographically and archaeologically in Australia's arid regions (Gould 1978:820). Roth (1897:101-2) recorded the manufacture and use of what he termed 'pot lid' flakes and 'native-gouge' composite adzes. In 1924, following their observations of the Wonkonguru people of the Lake Eyre region, Horne and Aiston (1924:80-9) recorded the use of a specific woodworking adze called 'koondi tuhla'. The 'tuhla', described as a broad semi-circular stone flake, was hafted to the end of a curved wooden handle or 'koondi' (the name meaning curved) with an organic fixative, such as spinifex (Triodia $\mathrm{sp}$ ) or beefwood (Grevillea sp) resin, and functioned generally as a chisel, scraper, axe and/or adze. The tuhla flake was periodically resharpened with a lightweight hammerstone or boomerang, and later unhafted and replaced when considered to be no longer of any use (Horne and Aiston 1924:89). Subsequent to Horne and Aiston's (1924, see also Aiston 1928, 1929) observations, the name of this tool was abbreviated to 'tula' (Figure 2).

Tulas were observed to have a variety of uses dependent on the dimensions of the flake, the hafting arrangement, and the amount of resharpening the flake sustained. Hafted tulas were 
Table 1. Results of residue and use-wear analyses of surface collected artefacts

\begin{tabular}{|c|c|c|c|c|c|c|}
\hline \multirow{2}{*}{$\begin{array}{l}\text { Tool } \\
\text { Type }\end{array}$} & \multirow{2}{*}{$\begin{array}{l}\text { Artefact } \\
\text { Number }\end{array}$} & \multicolumn{2}{|c|}{ Traces of Use-Wear } & \multicolumn{2}{|c|}{ Identified Residues } & \multirow[b]{2}{*}{$\begin{array}{l}\text { Suggested Task } \\
\text { Association }\end{array}$} \\
\hline & & $\begin{array}{l}\text { Dorsal } \\
\text { View }\end{array}$ & $\begin{array}{l}\text { Ventral } \\
\text { View }\end{array}$ & Dorsal View & Ventral View & \\
\hline Core & $999-53$ & $\begin{array}{l}\text { Lower } \\
\text { RLE - } \\
\text { retouch/ } \\
\text { use-wear } \\
\text { scars; } \\
\text { RLE - } \\
\text { fine } 60^{\circ} \& \\
45^{\circ} \text { striae; } \\
\text { OAR - } \\
\text { abraded } \\
\& \\
\text { rounded. }\end{array}$ & $\begin{array}{l}\text { Lower LLE } \\
\text { - retouch/ } \\
\text { use-wear } \\
\text { scars. }\end{array}$ & $\begin{array}{l}\text { OAR - Bone } \\
\text { collagen, opaque } \\
\text { resin; DA red/ } \\
\text { black resin; S - } \\
\text { plant cellulose \& } \\
\text { starch 2-4 } \mu \mathrm{m} \text {. }\end{array}$ & $\begin{array}{l}\text { DE - vivianite, red ochre; } \\
\text { DA - red/black resin; } S \text { - plant } \\
\text { cellulose \& starch } 2-4 \mu \mathrm{m} \text {. }\end{array}$ & $\begin{array}{l}\text { Bone- } \& \text { resinous } \\
\text { woodworking. }\end{array}$ \\
\hline \multirow[t]{5}{*}{ Blade } & $777-2(7)$ & $\begin{array}{l}\text { ROAR } \\
-45^{\circ} \\
\text { curved } \\
\text { striae \& } \\
\text { abraded. }\end{array}$ & $\begin{array}{l}\text { Adjacent } \\
\text { to PE - } 45^{\circ} \\
\text { drag marks } \\
\text { through } \\
\text { sediment. }\end{array}$ & $\begin{array}{l}\text { Edges and OAR } \\
\text { - opaque resin, } \\
\text { granular bone } \\
\text { collagen; RLE - } \\
\text { collagen fibril; S - } \\
\text { charcoal \& starch } \\
2-3 \mu \mathrm{m} \text {. }\end{array}$ & $\begin{array}{l}\text { RLE - hair; S - charcoal \& } \\
\text { starch 2-3 } \mu \mathrm{m} .\end{array}$ & $\begin{array}{l}\text { Bone- \& resinous } \\
\text { woodworking. }\end{array}$ \\
\hline & $\begin{array}{l}1600- \\
4(3)\end{array}$ & $\begin{array}{l}\text { OAR - } \\
\text { abraded; } \\
\text { DE, LLE, } \\
\text { RLE - } \\
\text { retouch/ } \\
\text { use-wear } \\
\text { scars. }\end{array}$ & $\begin{array}{l}\text { DE, LLE, } \\
\text { RLE - } \\
\text { retouch/use- } \\
\text { wear scars; } \\
\text { Upper LLE } \\
-45^{\circ} \text { drag } \\
\text { marks. }\end{array}$ & $\begin{array}{l}\text { PE - plant } \\
\text { cellulose; } \mathrm{S}- \\
\text { opaque, brown \& } \\
\text { red resin, starch } \\
2-4 \mu \mathrm{m} .\end{array}$ & $\begin{array}{l}\text { RLE - plant cellulose; LLE } \\
\text { - green resin; } \mathrm{S} \text { - opaque, } \\
\text { brown, \& red resin, starch } \\
2-4 \mu \mathrm{m} .\end{array}$ & $\begin{array}{l}\text { Resinous } \\
\text { woodworking. }\end{array}$ \\
\hline & $\begin{array}{l}7900- \\
2(1) \\
\left(T L^{*}\right)\end{array}$ & - & - & - & $\begin{array}{l}\text { Surface centre - nucleated } \\
\text { thin blood; Red ochre. }\end{array}$ & $\begin{array}{l}\text { Ritual activity, } \\
\text { male gender } \\
\text { (interpreted by } \\
\text { Ruby Saltmere, } \\
\text { Indjilandji Elder). }\end{array}$ \\
\hline & $\begin{array}{l}7900- \\
2(2) \\
\left(T^{*}\right)\end{array}$ & $\begin{array}{l}\text { RLE - } \\
\text { retouch/ } \\
\text { use-wear } \\
\text { scars. }\end{array}$ & $\begin{array}{l}\text { LLE - } \\
\text { retouch/use- } \\
\text { wear scars. }\end{array}$ & - & $\begin{array}{l}\text { LLE \& RLE - thin blood } \\
\text { smear with anucleate red } \\
\text { blood cells; S - downy and } \\
\text { pennaceous Chenonetta } \\
\text { jubata (wood duck) feather } \\
\text { fragments }\end{array}$ & $\begin{array}{l}\text { Ritual activity. } \\
\text { (interpreted by } \\
\text { Ruby Saltmere, } \\
\text { Indjilandji Elder). }\end{array}$ \\
\hline & $20700-4$ & $\begin{array}{l}\text { OAR - } \\
\text { abraded. }\end{array}$ & $\begin{array}{l}\text { None } \\
\text { observed. }\end{array}$ & $\begin{array}{l}\text { Adjacent to PE } \\
\text { - granular bone } \\
\text { collagen; PE, LLE } \\
\text { \& OAR - resin \& } \\
\text { plant tissue; S - } \\
\text { starch 2-3 } \mu \mathrm{m} \text {. }\end{array}$ & $\begin{array}{l}\text { Adjacent to PE - granular } \\
\text { bone collagen; } S \text { - starch 2-3 } \\
\mu \mathrm{m} \text {. }\end{array}$ & $\begin{array}{l}\text { Bone- \& plant } \\
\text { working. }\end{array}$ \\
\hline \multirow[t]{2}{*}{$\begin{array}{l}\text { Hand } \\
\text { Axe }\end{array}$} & $\begin{array}{l}999-67 \\
\left(T^{*}\right)\end{array}$ & $\begin{array}{l}\text { None } \\
\text { observed. }\end{array}$ & $\begin{array}{l}\text { Heavy } 25^{\circ} \\
\text { drag marks } \\
\text { away from } \\
\text { LLE. }\end{array}$ & $\begin{array}{l}\text { Lower LLE - plant } \\
\text { tissue \& ovate } \\
\text { starch up to } 6 \mu \mathrm{m} .\end{array}$ & $\begin{array}{l}\text { Lower LLE - plant tissue \& } \\
\text { ovate starch up to } 6 \mu \mathrm{m} .\end{array}$ & $\begin{array}{l}\text { Starchy plant } \\
\text { processing. }\end{array}$ \\
\hline & $\begin{array}{l}40400-2 \\
\left(T L^{*}\right)\end{array}$ & $\begin{array}{l}\text { None } \\
\text { observed. }\end{array}$ & $\begin{array}{l}\text { None } \\
\text { observed. }\end{array}$ & $\begin{array}{l}\text { Distal LLE - hair } \\
\& \text { collagen fibril } \\
\text { bundle. }\end{array}$ & - & $\begin{array}{l}\text { Late stage } \\
\text { butchery. }\end{array}$ \\
\hline \multirow[t]{2}{*}{ Tula } & $\begin{array}{l}21500-3 \\
\left(T^{*}\right)\end{array}$ & $\begin{array}{l}\text { Abraded } \\
\text { retouched } \\
\text { edges. } \\
\text { Distal } \\
\text { ridges - } \\
\text { striae } 20^{\circ} \\
\& 45^{\circ} .\end{array}$ & $\begin{array}{l}\text { Lower LLE } \\
-45^{\circ} \& 65^{\circ} \\
\text { striae. }\end{array}$ & - & $\begin{array}{l}1.2 \mathrm{~cm} \text { from DE }- \text { red/black \& } \\
\text { grey resin \& plant debris; Left } \\
D E-\text { woody tissue, abundant } \\
\text { plant cellulose, plastids with } \\
\text { starch \& resin. }\end{array}$ & $\begin{array}{l}\text { Once hafted; } \\
\text { Woodworking } \\
\text { \& starchy plant } \\
\text { processing. }\end{array}$ \\
\hline & $\begin{array}{l}23500-5 \\
\left(T^{*}\right)\end{array}$ & $\begin{array}{l}\text { Abraded } \\
\text { retouched } \\
\text { edges; } \\
\text { LLE - } \\
\text { vertical } \\
\text { dragging. }\end{array}$ & $\begin{array}{l}\text { Abraded } \\
\text { retouched } \\
\text { edges; DE } \\
-20^{\circ}, 45^{\circ} \\
60^{\circ} \& 90^{\circ} \\
\text { striae, } 60^{\circ} \\
\text { dragging. }\end{array}$ & $\begin{array}{l}\text { S - plant cellulose } \\
\& \text { starch } 1-2 \mu \mathrm{m} .\end{array}$ & $\begin{array}{l}\text { DE - red, yellow \& black } \\
\text { resin, DA - plant \& grass } \\
\text { fibres; } S \text { - starch } 1-2 \mu \mathrm{m} .\end{array}$ & $\begin{array}{l}\text { Woodworking } \\
\& \text { grass } \\
\text { processing. }\end{array}$ \\
\hline \multicolumn{7}{|c|}{$\begin{array}{l}\text { Key: } T L^{*} \text { - Dr Tom Loy limited field examination; } \\
\mathrm{DE} \text { - distal edge; } \mathrm{DA} \text { - distal area; LLE - left lateral edge; RLE - right lateral edge; OAR - obtuse angle ridge; ROAR } \\
\text { - right obtuse angle ridge; PE - platform edge; } \mathrm{S} \text { - scattered on surface; } \\
\mu \mathrm{m} \text { - micron (starch granule diameter). }\end{array}$} \\
\hline
\end{tabular}


Table 2. Results of residue and use-wear analyses of excavated artefacts

\begin{tabular}{|c|c|c|c|c|c|c|}
\hline \multirow{2}{*}{$\begin{array}{l}\text { Tool } \\
\text { Type }\end{array}$} & \multirow{2}{*}{$\begin{array}{l}\text { Artefact } \\
\text { Number }\end{array}$} & \multicolumn{2}{|c|}{ Traces of Use-Wear } & \multicolumn{2}{|c|}{ Identified Residues } & \multirow{2}{*}{$\begin{array}{l}\text { Suggested Task } \\
\text { Association }\end{array}$} \\
\hline & & Dorsal View & Ventral View & Dorsal View & Ventral View & \\
\hline \multirow[t]{3}{*}{ Core } & $16666-4$ & OARs - abraded. & $\begin{array}{l}\text { OARs - abraded; } \\
45^{\circ}, 60^{\circ} \text { and vertical } \\
\text { dragging on negative } \\
\text { scar. }\end{array}$ & $\mathrm{S}$ - starch $2-4 \mu \mathrm{m}$. & $\begin{array}{l}\text { Bone collagen on negative } \\
\text { scar edges; DA - wood } \\
\text { tissue; PE - opaque \& red/ } \\
\text { black resin; S - starch 2-4 } \\
\mu \mathrm{m} \text {. }\end{array}$ & $\begin{array}{l}\text { Once hafted; } \\
\text { Bone- \& woodworking. }\end{array}$ \\
\hline & $17666-4$ & Left PE - pitting. & $\begin{array}{l}\text { OAR - abraded; } \\
\text { Upper RLE - } 60^{\circ} \\
\text { dragging. }\end{array}$ & $\begin{array}{l}\text { S - opaque \& red/black resin, } \\
\text { charcoal \& starch } 2-4 \mu \mathrm{m} \text {. }\end{array}$ & $\begin{array}{l}\text { S - opaque \& red/black resin, } \\
\text { charcoal \& starch } 2-4 \mu \mathrm{m} \text {. }\end{array}$ & Woodworking. \\
\hline & $23666-11$ & $\begin{array}{l}\text { Retouch/use-wear } \\
\text { scars; Abraded } \\
\text { ridges; Right OAR } \\
\text { - rough \& pitted. }\end{array}$ & $\begin{array}{l}\text { OAR - abraded; } \\
\text { RLE - retouch/use- } \\
\text { wear scars; PE - } 45^{\circ} \\
\text { dragging. }\end{array}$ & $\begin{array}{l}\text { Opaque \& red/black resin } \\
\text { on ridges; Upper LOAR - } \\
\text { charcoal; S - plant tissue \& } \\
\text { starch } 2-4 \mu \mathrm{m} \text {. }\end{array}$ & $\begin{array}{l}\text { PE - plant exudate \& } \\
\text { charcoal; OARs - opaque \& } \\
\text { red/black resin on ridges; } \mathrm{S} \text { - } \\
\text { starch } 2-4 \mu \mathrm{m} \text {. }\end{array}$ & $\begin{array}{l}\text { Possibly once hafted; } \\
\text { Woodworking. }\end{array}$ \\
\hline \multirow[t]{6}{*}{ Blade } & $282-2$ & $\begin{array}{l}\text { LLE - retouch/use- } \\
\text { wear scars \& } 45^{\circ} \\
\text { striae; RLE - } 15^{\circ} \\
\text { to } 90^{\circ} \text { striae; OAR } \\
\text { - abraded with } 45^{\circ} \\
\text { striae either side. }\end{array}$ & $\begin{array}{l}\text { Lower LLE - vertical } \\
\text { striae; RLE - retouch/ } \\
\text { use-wear scars; } \\
\text { Lower RLE - } 45^{\circ} \& \\
90^{\circ} \text { drag marks. }\end{array}$ & $\begin{array}{l}\text { Edges - bone collagen; } \\
\text { Lower RLE \& DE - red } \\
\text { ochre; Upper RLE - feather } \\
\text { (Order - Galliformes); LLE } \\
\text { - plant tissue; S - opaque } \\
\text { \& red/black resin \& starch } \\
\text { 2-4 } \mu \mathrm{m} \text {. }\end{array}$ & $\begin{array}{l}\text { Edges - bone collagen; RLE, } \\
\text { LLE \& DE - red ochre; RLE } \\
\text { - plant tissue; S - opaque } \\
\& \text { red/black resin \& starch } \\
2-4 \mu \mathrm{m} \text {. }\end{array}$ & $\begin{array}{l}\text { Bone- } \& \text { woodworking } \& \\
\text { ceremonial use. }\end{array}$ \\
\hline & $4666-2(1)$ & $\begin{array}{l}\text { OAR - abraded; } \\
\text { LLE - retouch/ } \\
\text { use-wear scars \& } \\
\text { vertical striae; RLE } \\
\text {-vertical striae; DE } \\
\text { - almost vertical } \\
\text { striae. }\end{array}$ & $\begin{array}{l}\text { Upper LLE - } 10^{\circ} \\
\text { drag marks crossed } \\
\text { by } 45^{\circ} \text { striae; RLE } \\
\text { - retouch/use-wear } \\
\text { scars \& curved } 10^{\circ} \\
\text { striae; } \mathrm{DE} \text { - curved } \\
\text { almost vertical striae. }\end{array}$ & $\begin{array}{l}\text { OAR and all edges - } \\
\text { opaque, red/black, orange } \\
\text { \& black resin; LLE - hair; S } \\
\text { - starch } 1-3 \mu \mathrm{m} .\end{array}$ & $\begin{array}{l}\text { All edges - opaque, red/ } \\
\text { black, orange \& black resin; } \\
\text { RLE - plant tissue; S - starch } \\
1-3 \mu \mathrm{m} \text {. }\end{array}$ & $\begin{array}{l}\text { Possibly once hafted; } \\
\text { Woodworking. }\end{array}$ \\
\hline & $5666-2(1)$ & $\begin{array}{l}\text { OARs - abraded; } \\
\text { Upper LLE - } \\
\text { horizintal \& vertical } \\
\text { striae; Adjacent } \\
\text { to centre PE - } 15^{\circ} \\
\text { drag marks; Lower } \\
\text { RLE - } 60^{\circ} \text { striae \& } \\
\text { retouch/use-wear } \\
\text { scars. }\end{array}$ & $\begin{array}{l}\text { Adjacent to upper \& } \\
\text { lower LLE - vertical } \\
\text { dragging; PE - } \\
\text { horizontal dragging; } \\
\text { Upper RLE - 45 } \\
\text { striae; Lower RLE \& } \\
\text { Distal edge - vertical } \\
\text { striae. }\end{array}$ & $\begin{array}{l}\text { LOAR - starch } 6 \mu \mathrm{m} ; \mathrm{S} \text { - } \\
\text { opaque, red \& black resin, } \\
\text { charcoal \& starch } 2-4 \mu \mathrm{m} .\end{array}$ & $\begin{array}{l}\text { S - opaque, red \& black } \\
\text { resin, charcoal \& starch } \\
2-4 \mu \mathrm{m} \text {. }\end{array}$ & Woodworking. \\
\hline & $\begin{array}{l}14666- \\
2(2)\left(T^{*}\right)\end{array}$ & $\begin{array}{l}\text { OAR - abraded } \\
\text { with } 45^{\circ} \text { drag } \\
\text { marks to left; PE - } \\
\text { drag marks. }\end{array}$ & None observed. & $\begin{array}{l}\text { All edges - red ochre; LLE } \\
\& \mathrm{PE} \text { - opaque \& red/black } \\
\text { resin \& plant tissue; } \mathrm{S} \text { - } \\
\text { starch } 1-3 \mu \mathrm{m} \text {. }\end{array}$ & $\begin{array}{l}\mathrm{PE} \text { - red ochre; PE \& RLE } \\
\text { - opaque \& red/black resin; } \\
\mathrm{S} \text { - starch } 1-3 \mu \mathrm{m} .\end{array}$ & $\begin{array}{l}\text { Possibly once hafted; } \\
\text { Decorative/ ceremonial } \\
\text { use, bone- } \& \text { woodworking }\end{array}$ \\
\hline & $22666-2$ & $\begin{array}{l}\text { OAE - abraded; } \\
\text { Upper RLE \& PE } \\
-45^{\circ} \text { drag marks; } \\
\text { PE - } 90^{\circ} \text { drag } \\
\text { marks. }\end{array}$ & $\begin{array}{l}\text { LLE, RLE \& PE }-45^{\circ} \\
\text { crossed drag marks. }\end{array}$ & $\begin{array}{l}\text { LLE \& RLE - fibrous plant } \\
\text { tissue \& opaque resin; } \\
\text { Lower LLE - bone collagen } \\
\text { \& algal tissue; S - diatoms } \\
\text { (naviculoid, cymbelloid \& } \\
\text { elongate) \& starch } 1-2 \mu \mathrm{m} \text {. }\end{array}$ & $\begin{array}{l}\text { S - diatoms (naviculoid, } \\
\text { cymbelloid \& elongate) \& } \\
\text { starch } 1-2 \mu \mathrm{m} .\end{array}$ & Bone-working. \\
\hline & $\begin{array}{l}52666- \\
4(1)\left(T L^{*}\right)\end{array}$ & OAR - abraded. & $\begin{array}{l}\text { Worn bulb of } \\
\text { percussion. }\end{array}$ & S - fibrous plant tissue. & $\begin{array}{l}\text { Right half - fibrous plant } \\
\text { tissue; Upper RLE - } \\
\text { anuclear red blood cells. }\end{array}$ & Butchery. \\
\hline \multirow[t]{2}{*}{$\begin{array}{l}\text { Hand } \\
\text { Axe }\end{array}$} & $3666-5$ & $\begin{array}{l}\text { Some OARs } \\
\text { abraded, Mid } \\
\text { LLE - } 45^{\circ} \text { scraping; } \\
\text { LLE - abraded; } \\
\text { DE - } 90^{\circ} \text { striae \& } \\
\text { retouch/use-wear } \\
\text { scars. }\end{array}$ & $\begin{array}{l}\text { Some OARs } \\
\text { abraded; Lower RLE } \\
-90^{\circ} \text { curved drag } \\
\text { marks; DE - retouch/ } \\
\text { use-wear scars. }\end{array}$ & $\begin{array}{l}\text { LLE - plant tissue starch } \\
\text { average } 16 \mu \mathrm{m} \text {; RLE - plant } \\
\text { tissue, starch average } 12 \mu \mathrm{m} \text {; } \\
\mathrm{S} \text { - starch } 2-3 \mu \mathrm{m} \text {. }\end{array}$ & $\begin{array}{l}\text { LLE - plant tissue, starch } \\
\text { average } 18 \mu \mathrm{m} ; \mathrm{RLE}-\text { plant } \\
\text { tissue; DE - starch average } \\
6 \mu \mathrm{m} ; \mathrm{S} \text { - starch } 2-3 \mu \mathrm{m} .\end{array}$ & Starchy plant processing. \\
\hline & $\begin{array}{l}23666-12 \\
\left(T^{*}\right)\end{array}$ & OARs - abraded. & - & $\begin{array}{l}\text { Lower half - plant tissue; } \\
\text { DE - starch average } 6 \mu \mathrm{m} \\
\text { \& raphide. }\end{array}$ & - & Starchy plant processing. \\
\hline \multirow[t]{2}{*}{ Tula } & $\begin{array}{l}23666- \\
10(1)\end{array}$ & $\begin{array}{l}\text { OARs - abraded; } \\
\text { LLE - } 45^{\circ} \text { striae \& } \\
\text { crossing curved } \\
\text { rub marks; retouch/ } \\
\text { use-wear scars. }\end{array}$ & $\begin{array}{l}\text { Lower LLE - } 45^{\circ} \& \\
90^{\circ} \text { crossed striae; } \\
\text { RLE - } 60^{\circ} \& 90^{\circ} \\
\text { striae. }\end{array}$ & $\begin{array}{l}\text { All edges - bone collagen; } \\
\mathrm{S} \text { - wood \& plant tissue, } \\
\text { opaque \& red/black resin } \\
\text { patches \& starch } 2 \mu \mathrm{m} .\end{array}$ & $\begin{array}{l}\mathrm{PE} \text { - bone collagen; S - } \\
\text { wood \& plant tissue, opaque } \\
\& \text { red/black resin patches \& } \\
\text { starch } 2 \mu \mathrm{m} \text {. }\end{array}$ & Bone- and woodworking. \\
\hline & $\begin{array}{l}23666- \\
10(2) \\
\left(T L^{*}\right)\end{array}$ & - & $\begin{array}{l}\text { Retouch/use-wear } \\
\text { scars; Mid DE - } 45^{\circ} \\
\text { to } 60^{\circ} \text { heavy striae. }\end{array}$ & - & $\begin{array}{l}\text { All edges \& centre - plant } \\
\text { tissue; } \text { S - opaque \& yellow } \\
\text { resin \& starch average } 2 \mu \mathrm{m} .\end{array}$ & Woodworking. \\
\hline \multicolumn{7}{|c|}{$\begin{array}{l}\text { Key: } \\
\mathrm{TL}^{*} \text { - Dr Tom Loy limited field examination; } \\
\mathrm{DE} \text { - distal edge; } \mathrm{DA} \text { - distal area; LLE - left lateral edge; RLE - right lateral edge; OAR - obtuse angle ridge; ROAR - right obtuse angle ridge; LOAR - left obtuse angle } \\
\text { ridge; } \mathrm{PE} \text { - platform edge; } \\
\mathrm{S} \text { - scattered on surface; } \mu \mathrm{m} \text { - micron (starch granule diameter). }\end{array}$} \\
\hline
\end{tabular}

generally employed as woodworking tools for the manufacture of weapons, ceremonial and sacred objects and various other wooden implements such as water carriers, digging bowls and spearthrowers (Horne and Aiston 1924:103; McCarthy 1967:17,28; Roth 1904:17; Spencer and Gillen 1969:637). McCarthy (1967:28; see also Thomson 1964:418; Tindale 1965-1968:154) observed hafted tulas employed for butchering animals, bone working and occasional digging. Similarly, Etheridge (1891:41) described the use of unhafted tula flakes in skinning kangaroos, and Mitchell (1949:9) mentioned a hafted disc-shaped flake used for making notches in the bark of trees for climbing footholds. Davidson (1935:160) observed Aborigines in the Northern Territory using hafted tulas as throwing weapons and clubs. Gould et al. (1971:155) recorded that 

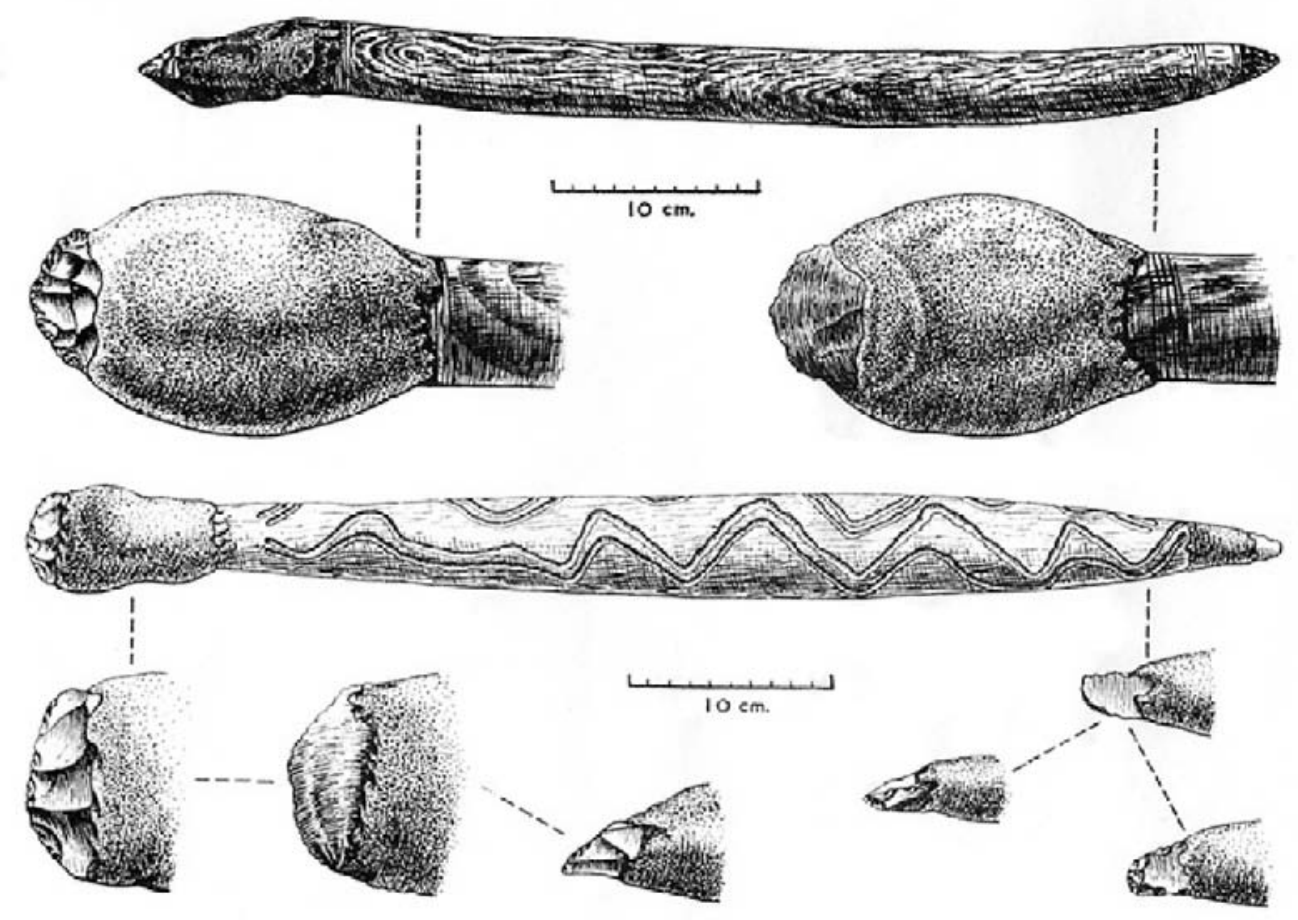

Figure 2. Illustration of hafted tula flakes employed by the Ngadadjara and Nakako people, South Australia (after Tindale 1965:134; courtesy Museum Board of South Australia).

the narrow edged flake, named 'pitjuru-pitjuru' by the Nyatunyatjara of the Western Desert, was employed exclusively for engraving decorations on sacred boards and spear-throwers.

During use, tula flakes were progressively resharpened along the distal/proximal extremity until they were too small to be hafted. At this point they were removed from the haft and either discarded, employed as hand scrapers and chisels, or rehafted to utilise other functional edges (McCarthy 1967:28-9). These 'worn-out' (Horne and Aiston 1924:89) flake remnants are commonly referred to as tula slugs (Campbell and Edwards 1966:161; Hiscock 1994:269; McCarthy 1967:28; McNiven 1993:23; Moore 2003:24).

\section{Methods of analysis and data collection}

Given the quantity of artefacts recovered, an analysis of the entire collected sample was beyond the scope of this study. Based on the notion that the individual artefact can be as informative as the larger site (Loy 1993:44), a sub-sample was examined with the aim of generating inferences about artefact use. Prior to the analysis, the tulas were divided into two morphologically defined categories of 'flake' and 'slug', based on size, shape and extent of retouch (see Table 3). From ethnographic accounts and current research into tula manufacture and use, it was expected that certain areas on the artefact's surface were more likely to contain residues than others. These areas guided the placement of the transect lines employed during the examination of each artefact, making a total of 512 analysed quadrants (Figure 3). The size of each quadrant depended on individual artefact morphology.

The data collection process was divided into macroscopic and microscopic examination, including low- and high-magnification (incident and transmitted light), and biochemical testing. During macroscopic examination, distinguishing features such as flake scars, fractures, and signs of retouch (as defined by Kamminga 1982:6) were noted. Low-magnification examination using the Wild microscope proceeded along the transect lines and the position, direction and quantity of residues were recorded, along with any signs of edge rounding (including bevelling), fractures, edge-chipping/scarring, striations, and polish. High-magnification incident microscopy was performed with an Olympus BX60. Residues were photographed and recorded in terms of colour, 
Table 3. Morphological and macroscopic examination of tulas

\begin{tabular}{|c|c|c|c|c|c|c|c|c|c|}
\hline \begin{tabular}{l|} 
Artefact \\
Number
\end{tabular} & \begin{tabular}{|c|} 
P L \\
$(\mathrm{mm})$
\end{tabular} & $\begin{array}{c}\text { P W } \\
(\mathrm{mm})\end{array}$ & $\begin{array}{c}\mathrm{TL} \\
(\mathrm{mm})\end{array}$ & $\begin{array}{c}\text { T W } \\
(\mathrm{mm})\end{array}$ & $\begin{array}{c}\mathrm{T} \mathrm{T} \\
(\mathrm{mm})\end{array}$ & $\begin{array}{l}\text { Dist. } \\
\text { Angle }\end{array}$ & $\begin{array}{c}\text { Prox.D } \\
\text { Angle }\end{array}$ & $\begin{array}{l}\text { Prox.V } \\
\text { Angle }\end{array}$ & Artefact Description \\
\hline \begin{tabular}{|l|}
$20100-7 a$ \\
(Flake)
\end{tabular} & 35 & 7 & 27 & 33 & 7 & $60^{\circ}$ & $60^{\circ}$ & $120^{\circ}$ & $\begin{array}{l}\text { DF Flake scars along left lateral margin to point at distal edge, same for opposite margin: VF } \\
\text { Eraillure scar on bulb of percussion, two feather fractures on right dorsal edge of platform, } \\
\text { fine edge chipping along left lateral margin. }\end{array}$ \\
\hline $\begin{array}{l}22300-4 \\
\text { (Flake) }\end{array}$ & 42 & 11 & 30 & 48 & 11 & $45^{\circ}$ & $45^{\circ}$ & $120^{\circ}$ & $\begin{array}{l}\text { DF Flake scars from platform overhang removal, flake scars along left, right and distal edges: } \\
\text { VF Small step and feather fractures on distal edge. }\end{array}$ \\
\hline $\begin{array}{l}999.428 \\
\text { (Flake) }\end{array}$ & 31 & 11 & 27.5 & 38 & 7 & $45^{\circ}$ & $45^{\circ}$ & $120^{\circ}$ & $\begin{array}{l}\text { DF Feather fractures along platform edge, flake removal scars along left, right and distal } \\
\text { edges: VF Large feather fracture originating from left dorsal edge on platform, edge chipping } \\
\text { on left, right and distal edges. }\end{array}$ \\
\hline $\begin{array}{l}34700-3 \\
\text { (Flake) }\end{array}$ & 41.5 & 8.5 & 28.5 & 49 & 13.5 & $45^{\circ}$ & $85^{\circ}$ & $127.5^{\circ}$ & $\begin{array}{l}\text { DF Large flake removal scar on right platform edge, step fractures along right lateral margin, } \\
\text { large step fractures along distal edge with edge chipping: VF Large feather fracture on left } \\
\text { lateral margin and distal edge, edge chipping along dorsal edge of platform. }\end{array}$ \\
\hline $\begin{array}{ll}66800-3 \\
\text { (Flake) }\end{array}$ & 34 & 9.5 & 34 & 35 & 9 & $60^{\circ}$ & $60^{\circ}$ & $120^{\circ}$ & $\begin{array}{l}\text { DF Overhang removal along platform edge with two large step fractures on left and right } \\
\text { sides, flake removal scars along left, right and distal edges, moderate fracturing along right } \\
\text { lateral margin: VF "6680-3" written in pencil along bulb of percussion. }\end{array}$ \\
\hline \begin{tabular}{|l|}
$60400-2$ \\
(Flake)
\end{tabular} & 42 & 18 & 31 & 43 & 9.5 & $45^{\circ}$ & $60^{\circ}$ & $127.5^{\circ}$ & $\begin{array}{l}\text { DF Large obtuse angle ridge upper left lateral margin to lower right lateral margin, large } \\
\text { step fracture left platform edge, chipping along left and distal edges, concave edge lower } \\
\text { right lateral margin: VF Step fractures along right lateral margin, eraillure scar on bulb of } \\
\text { percussion, edge chipping along distal edge. }\end{array}$ \\
\hline $\begin{array}{l}\text { 20100-7b } \\
\text { (Flake) }\end{array}$ & 31 & 11 & 40 & 47.5 & 10.5 & $60^{\circ}$ & $60^{\circ}$ & $120^{\circ}$ & $\begin{array}{l}\text { DF Small step fractures middle of platform edge, concave edge lower right lateral margin to } \\
\text { distal edge: VF Edge chipping upper left lateral margin, step fractures along distal edge, two } \\
\text { feather fractures on dorsal edge of platform. }\end{array}$ \\
\hline $\begin{array}{l}1100-4 a \\
\text { (Flake) }\end{array}$ & 36 & 11 & 27 & 43 & 9 & $60^{\circ}$ & $45^{\circ}$ & $127.5^{\circ}$ & $\begin{array}{l}\text { DF Edge chipping and step fractures along platform edge, flake removal scars along left, } \\
\text { right and distal edges: VF Feather fracture on distal edge, compression rings along bulb of } \\
\text { percussion, feather fractures on upper left lateral edge, two large feather fractures on dorsal } \\
\text { edge of platform. }\end{array}$ \\
\hline $\begin{array}{l}777-7 \\
\text { (Flake) }\end{array}$ & 40 & 11.5 & 26.5 & 42 & 10 & $60^{\circ}$ & $45^{\circ}$ & $120^{\circ}$ & $\begin{array}{l}\text { DF Edge chipping along platform edge, large flake removal scars along left and right lateral } \\
\text { margins and along distal edge, obtuse angle ridge from upper right lateral margin to lower left } \\
\text { lateral margin: VF Slight edge chipping on dorsal edge of platform, slight edge chipping along } \\
\text { lower left lateral margin and distal edge. }\end{array}$ \\
\hline $\begin{array}{l}1600-3 \\
\text { (Flake) }\end{array}$ & 24 & 6.5 & 22.5 & 28.5 & 7 & $52.5^{\circ}$ & $60^{\circ}$ & $105^{\circ}$ & $\begin{array}{l}\text { DF Slight chipping along platform edge, large feather and step fractures upper right lateral } \\
\text { margin, flake removal scars along left and right lateral margins and along distal edge: VF } \\
\text { Large feather fracture from distal edge across face, flake removal scars below right platform } \\
\text { edge, edge chipping along distal edge. }\end{array}$ \\
\hline $\begin{array}{l}1100-5 \\
\text { (Flake) }\end{array}$ & 35 & 9 & 27.5 & 38 & 9 & $60^{\circ}$ & $67.5^{\circ}$ & $112.5^{\circ}$ & $\begin{array}{l}\text { DF Feather fractures along platform edge, large feather fracture on right platform edge, flake } \\
\text { removal scars along distal edge and left lateral margin: VF Slight edge chipping along left } \\
\text { lateral margin and distal edge, small step fracture upper left lateral margin. }\end{array}$ \\
\hline $\begin{array}{l}62600-1 \\
\text { (Flake) }\end{array}$ & 34 & 9.5 & 29.5 & 38 & 9.5 & $45^{\circ}$ & $60^{\circ}$ & $120^{\circ}$ & $\begin{array}{l}\text { DF Large step fracture along platform edge, large concave flake removal on right lateral } \\
\text { edge, flake removal scars along left and distal edges, obtuse angle ridge above distal edge: } \\
\text { VF Edge chipping along right and distal edges, compression rings along bulb of percussion, } \\
\text { feather and step fractures along platform. }\end{array}$ \\
\hline $\begin{array}{l}30900-2 \\
\text { (Slug) }\end{array}$ & 40 & 9 & 21.5 & 47 & 12 & $75^{\circ}$ & $60^{\circ}$ & $125.5^{\circ}$ & $\begin{array}{l}\text { DF Multiple retroflexed hinge flake scars along distal edge, overhang removal and large } \\
\text { feather fractures along platform edge: VF Feather fracture on dorsal edge of platform, feather } \\
\text { fracture on left lateral margin, slight edge chipping on right lateral margin. }\end{array}$ \\
\hline $\begin{array}{l}31800-1 \\
\text { (Slug) }\end{array}$ & 41 & 11.5 & 17.5 & 45 & 11 & $75^{\circ}$ & $60^{\circ}$ & $97.5^{\circ}$ & $\begin{array}{l}\text { DF Edge chipping and feather fracture along platform edge, multiple retroflexed hinge flake } \\
\text { scars along distal edge, slight edge chipping along left and right lateral margins: VF Slight } \\
\text { edge chipping and edge fracture along distal edge, slight edge chipping along left lateral } \\
\text { margin. }\end{array}$ \\
\hline $\begin{array}{l}1100-4 b \\
\text { (Slug) }\end{array}$ & 35 & 10 & 17 & 40 & 10 & $75^{\circ}$ & $75^{\circ}$ & $97.5^{\circ}$ & $\begin{array}{l}\text { DF Multiple retroflexed hinge flake scars along distal edge, overhang removal and step } \\
\text { fractures along platform edge, flake scar along right lateral edge: VF Edge chipping along } \\
\text { distal edge. }\end{array}$ \\
\hline \begin{tabular}{|l|}
$\begin{array}{l}9800-4 \\
\text { (Slug) }\end{array}$ \\
\end{tabular} & 30 & 8 & 15.5 & 37 & 8 & $82.5^{\circ}$ & $67.5^{\circ}$ & $120^{\circ}$ & $\begin{array}{l}\text { DF Step fractures and edge chipping along platform edge, multiple retroflexed hinge flake } \\
\text { scars along distal edge: VF Edge chipping along distal edge. }\end{array}$ \\
\hline
\end{tabular}




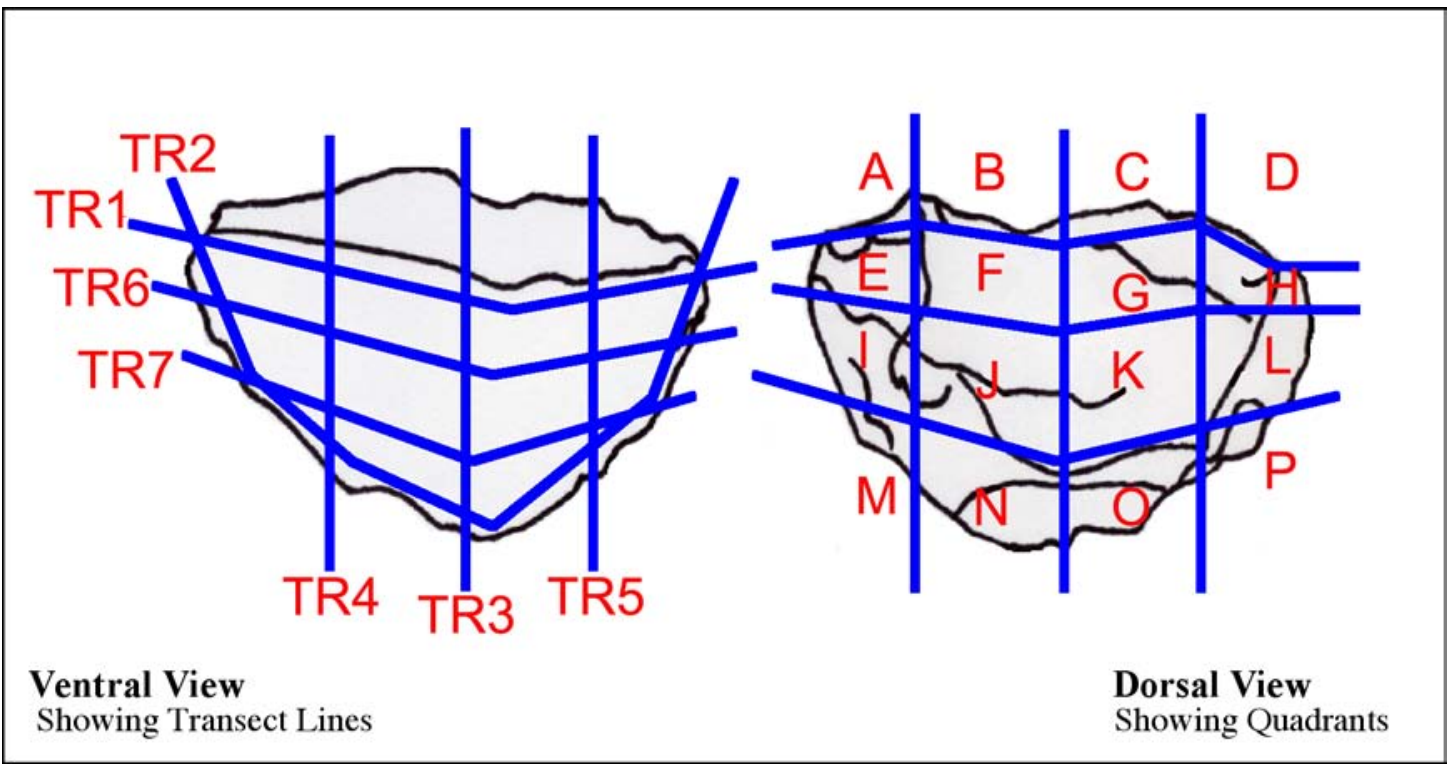

Figure 3. Detail of transect lines and quadrants used in analysis.

form, texture, identifiable structures, and amount (small, moderate and large) on the artefact surface. Striations, edge rounding, fractures and polish were also recorded and photographed. Residues requiring further examination were removed and placed on microslides and viewed using an Olympus BX50 polarising microscope. A reference collection of plant and animal residues specific to Australian flora and fauna, the Laboratory Microscopy guide (Archaeological Sciences Laboratory, University of Queensland), illustrated literature of plant and animal tissues, the Hemastix colourimetric test (Loy 1983) and samples collected from the site assisted in the identification of observed residues.

\section{RESULTS}

\section{Macroscopic examination}

With the exception of size and curvature of the distal edges, all tulas in the sample have distinct morphological similarities (Table 3). Within the 'flake' category, moderate to extensive flake scarring was identified along all edges (excluding the ventral platform edge), predominantly of step and hinge termination types. Other observations included: fine edge retouch along the dorsal side of the proximal edge and some degree of fine lateral and distal retouch; a smooth convex ventral surface (excluding artefacts 20100-7 and 60400-2, which have errailure scars along the bulb of percussion); a maximum width greater than the maximum length; an acute distal edge and dorsal platform edge angle; and an obtuse ventral platform edge angle. The tula 'slugs', although possessing the same morphological attributes, have additional characteristics dissimilar to the tula 'flakes'. These were observed as: a marked reduction in the length of the flake; a straight or slightly convex distal edge; large feather and step flake scars along dorsal platform edges (excluding artefact 31800-1, which has minimal retouch along this edge); and multiple flake scarring along the distal edge on the dorsal face, particularly of hinge and retroflexed hinge types.

\section{Low-magnification examination}

All of the tulas display some degree of microfracture scarring and rounding along the distal, lateral and dorsal platform edges. The most frequently occurring wear types observed were micro step, feather and hinge termination scars, edge rounding and micro edge-chipping (Figure 4). The term edge-chipping was applied to consecutive indiscriminate microfractures, occasionally of a rounded or angular morphology, as the individual flake scars were non-diagnostic of any of the above mentioned termination types. Edge-chipping constitutes the most prominent wear attribute, occurring in varying degrees on all artefacts in the sample. Step fracturing was also observed 

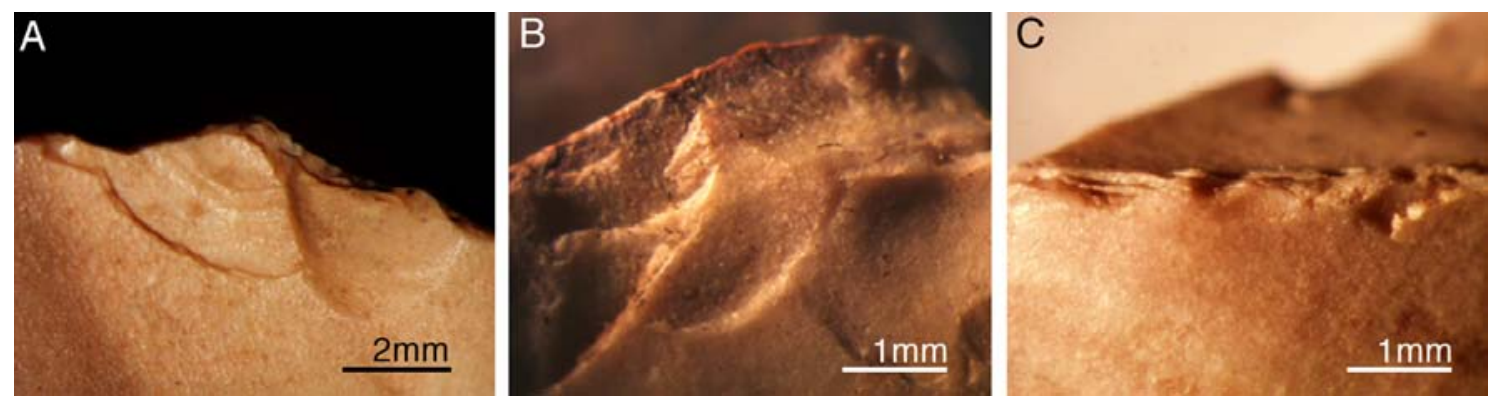

Figure 4. Examples of step, feather and hinge termination scars, and micro edge chipping. (A) Step and feather fractures. Artefact 1100-5, ventral right lateral margin, 8x (B) Multiple step and hinge fractures. Artefact 20100-7b, ventral distal edge, 16x (C) Edge chipping. Artefact 20100-7a, ventral left lateral margin, 16x.

on all artefacts (excluding 1100-5), predominantly on the distal edges, and the dorsal left lateral margin, although 20100-7a, 1600-3 and 30900-2 displayed step fracturing proximal to the bulb of percussion.

Although edge rounding was common it was usually restricted to the smoothing of microfractures along the perimeter edges. Some degree of edge rounding is also present on the obtuse angle ridges on artefacts 20100-7a, 60400-2, 1100-4a and 30900-2. Striations, surface polish, bending fractures, and compression ring fractures were observed relatively infrequently on only a few artefacts at low magnifications. All tulas in the sample have varying amounts of sediment and other residue adhering to their surfaces. Seven tula flakes and three slugs have plant fibres attached to their surfaces, which are probably an environmental additive rather than use-related. Various other residues were observed at low magnifications such as red resinous exudate and bone collagen (later identified during high-magnification microscopy) (Figure 5). Black fungal spores were also identified on some of the artefacts.

\section{High-magnification examination}

During high-magnification analysis, four types of use-wear were observed and a total of 37 individual residue types were identified across the sample. Suspected blood residues were located on the ventral face of six artefacts (22300-4, 34700-3, 60400-2, 777-7 and 31800-1 and on both faces of artefact 1100-4a). These deposits occur as either thin, colourless plaques with raised cracking or as dark reddish/brown cracked masses (Figure 6A). Blood films were occasionally observed in association with deposits of bone collagen (Figure 6B). Bone collagen was the most frequently observed animal residue, occurring in varying amounts on all artefacts in the sample (with the exception of 1100-4b). Artefact 31800-1 displays the largest occurrence of collagen on both the ventral and dorsal faces. Collagen, identified primarily as white, granular smears or fragments (Figure 6C) but also as translucent/grey amorphous sheets with a distinctive 'basketweave' appearance (Figure 7A), was occasionally found in the presence of vivianite (see below). Feather fragments are present on five of the tulas in small and moderate quantities, although no diagnostic downy barbules were identified (Figure 7B). Degraded hair fragments, often embedded in resin, were also observed (Figure 7C).

Plant residues consist of tissue, cells, cellulose, fibres, exudate, phytoliths, raphides, and pollen. These residues were observed predominantly in association with each other, occurring in moderate to large quantities on many of the tulas. Cellulose, of varying amounts, was the most common plant residue type observed across the sample and when found in association with other plant tissue components, such as vessel elements, bordered pits, wall thickenings and tracheids, was assumed a derivative of the associated plant material. Large deposits of plant tissue frequently occur as inclusions in resin or are caught beneath micro fractures and along edges. Occasionally, plant tissue was observed with the dermal and ground cellular structure intact. These tissue cells, located on artefacts 20100-7a, 22300-4, 66800-3, 62600-1, 30900-2, 31800-1 and 9800-4 are similar in structure to the epidermis and parenchyma of woody plants (Raven et al. 1999:586) 

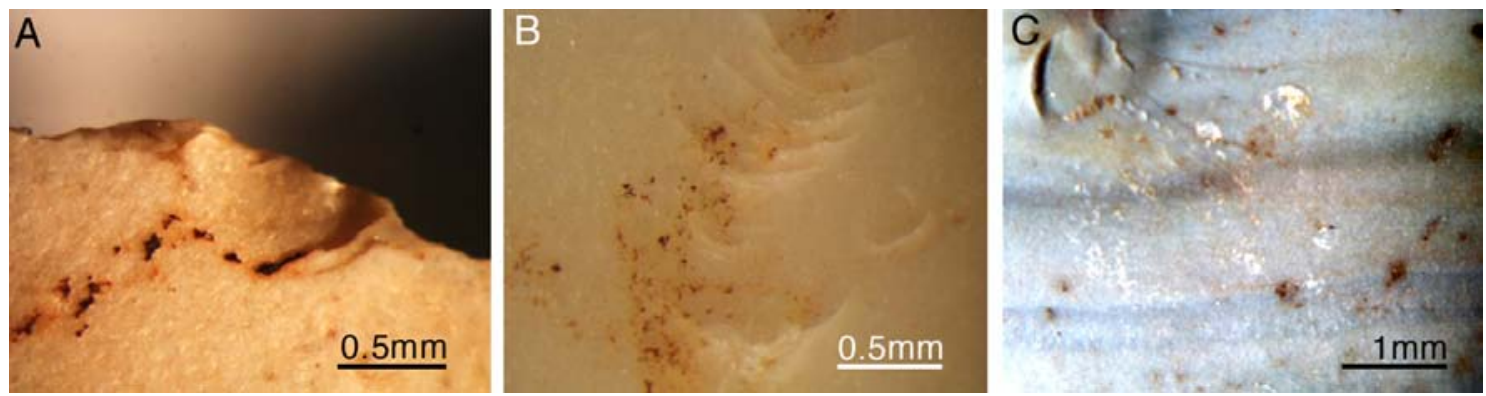

Figure 5. Residue in association with signs of use-wear. (A) Step fracture with resin. Artefact 60400-2, ventral distal edge, 25x. (B) Resin and compression ring fractures. Artefact 11004a, ventral mid section, 25x. (C) Step fracture, resin and bone collagen. Artefact 34700-3, ventral mid section, $16 x$.
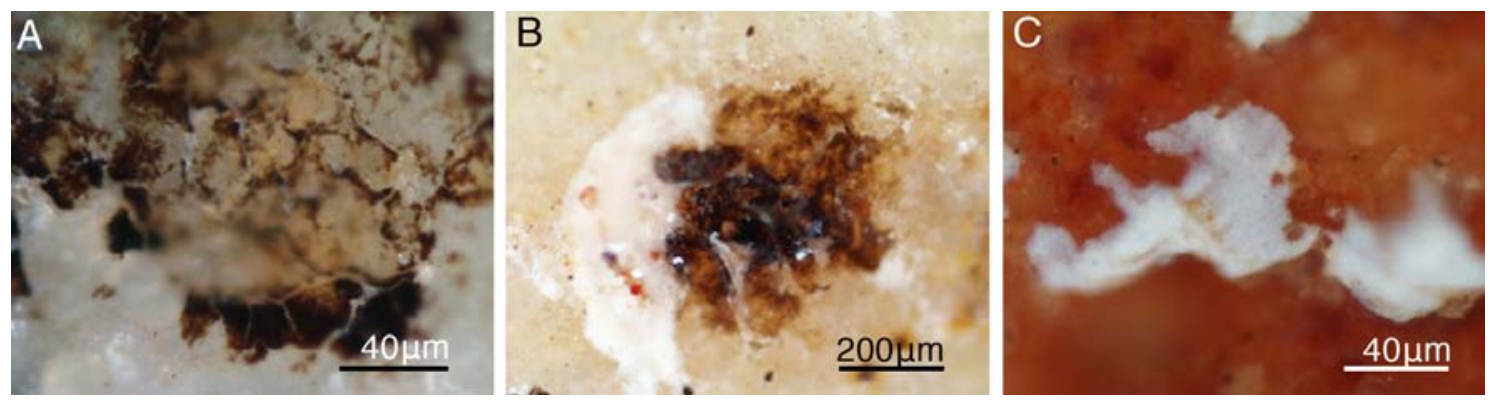

Figure 6. (A) Blood residue. Artefact 60400-2, right mid section ventral face, 500x bright field illumination, cross-polarised (BF xpol.). (B) Blood and collagen residue. Artefact 22300-4, distal edge ventral face, 100x BF xpol. (C) Granular bone collagen. Artefact 999.428, left platform ventral face, 500x BF xpol.
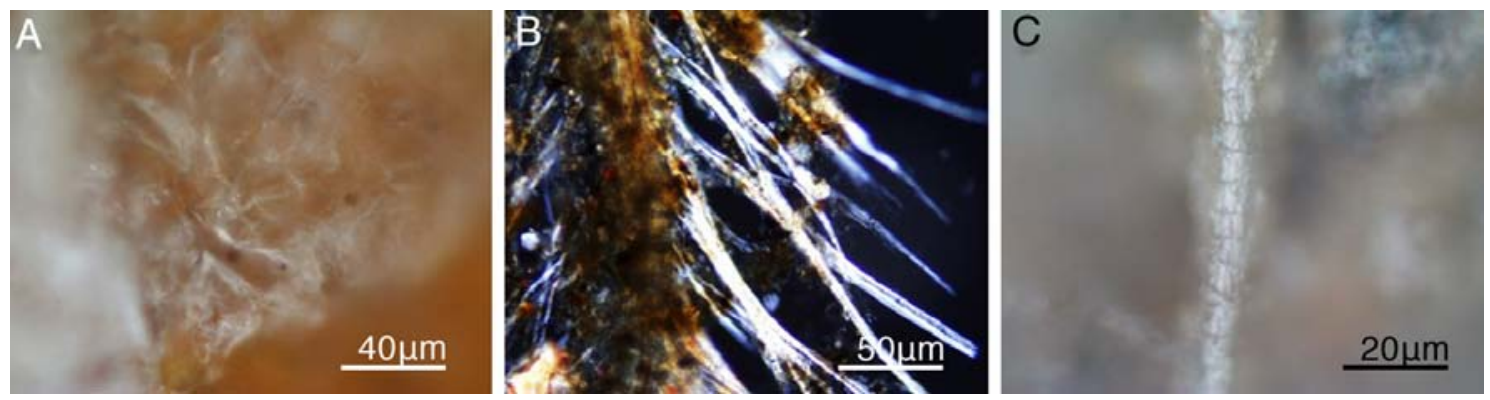

Figure 7. (A) Sheet collagen. Artefact 30900-2, middle left lateral margin dorsal face, 500x brightfield illumination plane-polarised (BF ppol.). (B) Feather barb. Artefact 62600-1, middle left lateral margin ventral face, 400x BF ppol (transmitted light). (C) Degraded hair. Artefact 62600-1, upper right lateral margin ventral face, 1000x BF ppol.

(Figure 8A). Plant exudate or sap (produced in vacuoles) was also observed in conjunction with plant tissue, occurring as colourless, glossy films, although generally in small quantities (Raven, et al. 1999:173). Phytoliths, primarily of festucoid and panicoid types, were observed on many of the tulas either individually or in rows within plant tissue (Figure 8B). Dumbbell phytoliths range in size from $15 \mu \mathrm{m}$ to $25 \mu \mathrm{m}$ in length, while ovate phytoliths vary in diameter from $10 \mu \mathrm{m}$ to $15 \mu \mathrm{m}$. Bundles of whisker raphides were observed on the edges of artefact 34700-3 (Figure 8C) and on the ventral right lateral margin of artefact 66800-3. Clusters of pollen were identified on the upper left lateral margin of artefact 30900-2, and a few scattered pollen grains were observed on the dorsal face of artefacts 20100-7a and 20100-7b. Starch grains (1-4 $\mu \mathrm{m}$ diameter), some of which are damaged or gelatinised, were observed on the majority of tulas.

Resin was the most frequently observed residue type, occurring in varying quantities on all tulas. The types of resin exhibited are red, orange/amber, clear/grey and charred. The most 

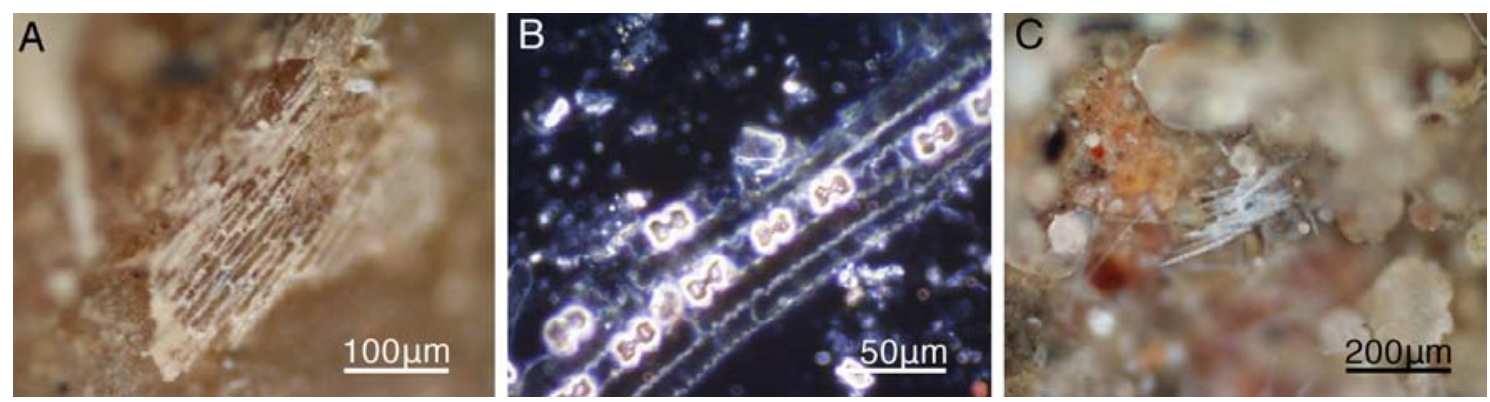

Figure 8. (A) Woody plant tissue. Artefact 22300-4, left platform edge ventral face, 200x dark field illumniatoin (DF). (B) Festucoid grass phytoliths. Artefact 22300-4, distal edge ventral face, 400x DF (transmitted light). (C) Bundle of raphides. Artefact 34700-3, distal edge dorsal face, 100x BF xpol.

consistently occurring type is red resin, predominantly observed in moderate and large quantities on both the dorsal and ventral faces of all artefacts except 9800-4. Red resin appeared as globular masses or smears and is generally located perpendicular to an edge, caught in microfractures, or along ridges (Figure 9A). Charred resin, regularly observed in association with red resin, is similar to the latter in appearance but is birefringent in plane-polarised light due to a high charcoal content. The physical similarities and location suggest charred resin is probably a carbonised form of red resin. Clear/grey resin, observed in similar frequency to red resin, appears either as thin grey smears or thick deposits containing a high proportion of grass phytoliths in addition to other plant material such as fibres and trichomes (plant hairs) (Figure 9B). Orange/amber resin with its distinct translucent, cracked morphology (Figure 9C) was observed in small and moderate quantities on the majority of the tulas.

Charcoal was observed primarily in association with resin (particularly charred resin) and various plant residues on all tulas. Charcoal appeared either scraped onto the surface in linear deposits or as individual fragments, with the largest quantities occurring on the dorsal faces of artefacts 20100-7a, 999.428 and 62600-1, and on the ventral face of artefacts 66800-3 and 16003. Both red and yellow ochre (Figure 10A) were also found on the majority of the tulas, with the largest deposits occurring predominantly toward the lower mid section and distal edges of artefact 22300-4 and 1600-3.

Possible post-use residues, or environmental additives were identified in varying quantities on both the ventral and dorsal faces of the majority of tulas. These include plant tissue, algae, fungal spores and hyphae, pennate (freshwater) diatoms, insect remains, including spidermite webs, eggs, wings and legs, silt, mineral crystals (possibly calcium oxalate), spherulites (calcium carbonate crystals often formed in avian and reptile uric acid) (Canti 1998:442), and graphite (pencil).

Use-wear identified at high-magnifications included striations, edge rounding, fractures, and surface polish. Striations were commonly observed either through residue, such as resin and bone collagen (Figure 10B), or directly on the stone surface. The latter striations were generally noted as fine, multidirectional and intersecting and probably formed as a result of soil abrasion (Kamminga 1982:14). Conversely, striations through residue are generally unidirectional and indicate either how the residue was applied to the surface or the subsequent direction of use. Edge rounding or smoothing occur largely along the distal edges of most tulas and along the dorsal obtuse angle ridge of 34700-3 and 777-7. Edge rounding was often observed with striations perpendicular or sub-perpendicular to the edge (between $65^{\circ}$ and $80^{\circ}$ ), and with some degree of surface polish. Surface polish or abrasion was identified as a marked increase in surface shine and occur in pitted and interlocking patches along surface protrusions and/or parallel to an edge (Figure 10C). All forms of use-wear increase in intensity towards the distal edges, with some degree of wear observable along the platform edges and the upper lateral margins. A summary of the residues and use-wear observed per artefact is presented in Table 4. 

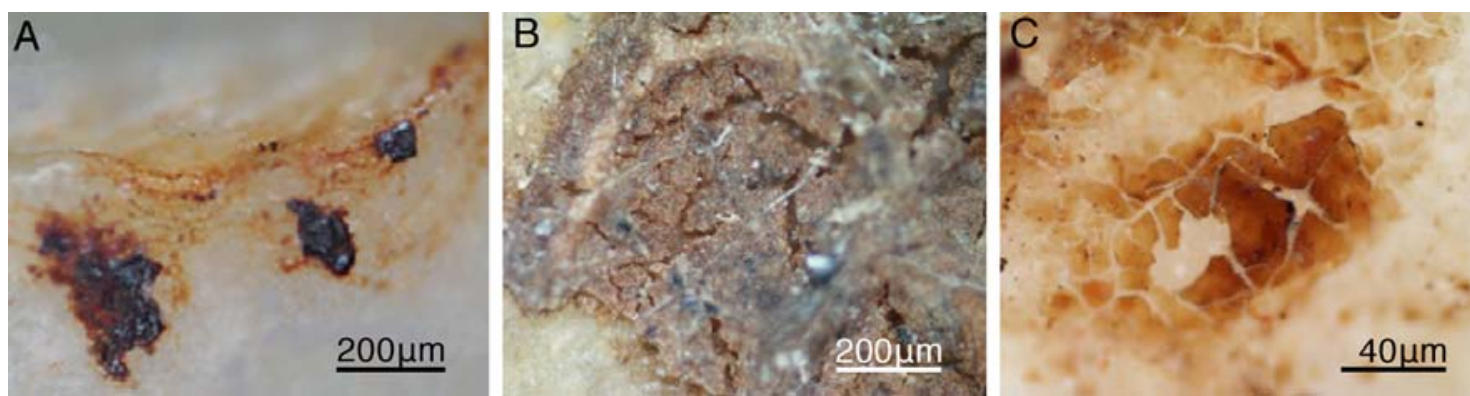

Figure 9. (A) Red resin along ridge. Artefact $66800-3$, right lateral margin dorsal face, $100 x$ BF xpol. (B) Mass of clear/grey resin. Artefact 22300-4, upper left lateral margin dorsal face, 100x DF. (C) Orange/amber resin. Artefact 34700-3, upper platform edge dorsal face, 500x BF xpol.
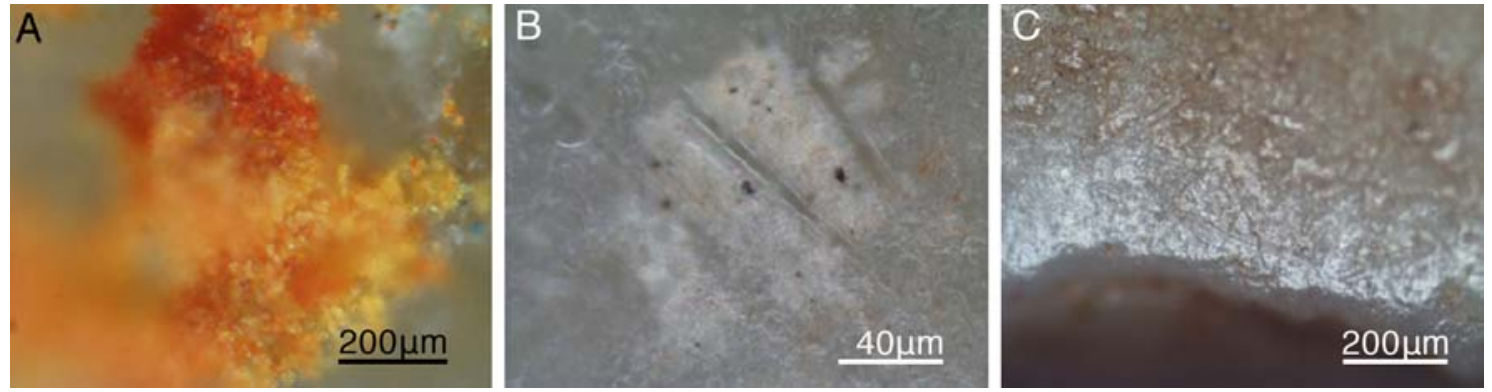

Figure 10. (A) Red and yellow ochre. Artefact 22300-4, distal edge ventral face, 100x BF xpol. (B) Striations through bone collagen.Artefact 31800-1, right lateral margin dorsal face, 500x DF. (C) Edge polish and striations. Artefact 66800-3, distal edge dorsal face, 200x BF ppol.

Subsequent to high-magnification microscopy, six samples of suspected blood residue were removed and tested using the Hemastix colourimetric test for the presence of hemoglobin or myoglobin. Three of the six returned a positive reaction prior to the addition of sodium EDTA (a chelating agent that removes chlorophyll and/or heavy metals that may also react positively). Artefact 22300-4 scored the highest concentration reaction of five (equivalent to three nanograms of blood per microlitre), and with the addition of the chelating agent returned a result of three ( 0.5 nanograms per microlitre). Artefact 60400-2 scored a reaction of three and with sodium EDTA, scored a trace reaction. The sample from 1100-4a scored an initial trace reaction but failed to produce a reaction with the addition of sodium EDTA.

\section{ANALYSIS AND DISCUSSION}

Taking into consideration the unknown conditions of the open site and the variety and extent of environmental additives, the presence of a particular residue such as plant tissue alone was not considered sufficient to suggest task association. Based on the various combinations of residues observed on the tulas and in further consideration of previous residue analyses of archaeological, ethnographic and replicated tools, a list of common residue types and the associated tasks from which they were produced was formulated (Table 5). A particular task was inferred if the combination of identified residues on the artefacts was consistent with the residue types presented in this table.

\section{Residues}

The most commonly occurring residues (excluding clear/grey resin) identified on both the dorsal and ventral sides of the tulas were bone collagen, charcoal, red resin, and woody plant tissue. The combination of the latter two residues suggests that working resinous wood was a consistent task 
Table 4. Summary of the quantities of use-related residue and wear observed at high magnification

\begin{tabular}{|c|c|c|c|c|c|c|c|c|c|c|c|c|c|c|c|c|}
\hline \multirow[b]{2}{*}{$\begin{array}{l}\text { Identified } \\
\text { Residues } \\
\end{array}$} & \multicolumn{2}{|c|}{$20100-7 a$} & \multicolumn{2}{|c|}{$22300-4$} & \multicolumn{2}{|c|}{999.428} & \multicolumn{2}{|c|}{$34700-3$} & \multicolumn{2}{|c|}{$66800-3$} & \multicolumn{2}{|c|}{$60400-2$} & \multicolumn{2}{|c|}{$20100-7 b$} & \multicolumn{2}{|c|}{$1100-4 a$} \\
\hline & D & v & D & $\mathbf{v}$ & D & v & D & $\mathbf{v}$ & D & v & D & $\mathbf{v}$ & D & $\mathbf{v}$ & D & v \\
\hline Blood & - & - & - & L & - & - & - & M & - & - & - & $S$ & - & - & $\mathrm{S}$ & $\mathrm{s}$ \\
\hline Collagen & $\mathrm{M}$ & - & $S$ & L & M & $\mathrm{L}$ & $S$ & L & $S$ & L & $\mathrm{M}$ & $\mathrm{M}$ & M & L & M & M \\
\hline Vivianite & - & - & - & - & - & $S$ & - & $\mathrm{S}$ & - & $S$ & - & - & - & S & - & - \\
\hline Feather & - & - & - & - & M & - & - & - & - & - & - & - & - & $\mathrm{S}$ & - & - \\
\hline Hair & $S$ & - & - & - & - & $S$ & $S$ & - & - & S & $\mathrm{S}$ & - & - & - & - & - \\
\hline Plant Tissue & L & - & $\mathrm{L}$ & $\mathrm{M}$ & L & M & L & $S$ & - & L & $\mathrm{M}$ & - & - & $\mathrm{S}$ & - & - \\
\hline Cellulose & L & $\mathrm{M}$ & $\mathrm{M}$ & $S$ & $M$ & $S$ & M & M & $S$ & $M$ & $S$ & $S$ & $\mathrm{~S}$ & $S$ & $\mathrm{~S}$ & - \\
\hline Phytoliths & - & - & $S$ & $\mathrm{~S}$ & $S$ & - & - & - & - & M & $S$ & - & - & - & $\mathrm{s}$ & - \\
\hline Raphides & - & - & - & - & - & - & L & $\mathrm{S}$ & - & $\mathrm{S}$ & - & - & - & - & - & - \\
\hline Starch Granules & SG & - & SG & ST & - & - & MTG & ST & - & LTG & SG & - & SG & SG & - & - \\
\hline Red Resin & $\mathrm{L}$ & $\mathrm{M}$ & $\mathrm{L}$ & $\mathrm{L}$ & $M$ & $\mathrm{~L}$ & L & $L$ & $\mathrm{~L}$ & L & $\mathrm{M}$ & $L$ & $M$ & $\mathrm{M}$ & L & $\mathrm{L}$ \\
\hline $\begin{array}{l}\text { Orange/Amber } \\
\text { Resin }\end{array}$ & - & $\mathrm{S}$ & $S$ & $\mathrm{~S}$ & $S$ & M & M & $\mathrm{s}$ & $S$ & $\mathrm{~S}$ & M & $S$ & $\mathrm{~s}$ & $\mathrm{M}$ & M & $\mathrm{s}$ \\
\hline Clear/Grey Resin & - & - & $\mathrm{M}$ & $\mathrm{S}$ & M & L & M & L & $S$ & L & L & $S$ & $\mathrm{~s}$ & S & $\mathrm{S}$ & $\mathrm{L}$ \\
\hline Charred Resin & $\mathrm{L}$ & $\mathrm{S}$ & $\mathrm{L}$ & M & $S$ & $\mathrm{~S}$ & M & $\mathrm{s}$ & $\mathrm{S}$ & M & - & $\mathrm{L}$ & M & - & $\mathrm{S}$ & $\mathrm{s}$ \\
\hline Charcoal & $\mathrm{L}$ & $\mathrm{M}$ & $S$ & $\mathrm{~S}$ & L & $S$ & $\mathrm{~S}$ & M & $\mathrm{S}$ & L & $S$ & M & M & $\mathrm{S}$ & $\mathrm{S}$ & - \\
\hline Ochre & $\mathrm{M}$ & - & $S$ & $\mathrm{~S}$ & $S$ & - & - & - & - & $S$ & - & $S$ & $\mathrm{~S}$ & - & $\mathrm{S}$ & - \\
\hline Diatoms & S & - & - & - & - & - & - & - & - & - & - & $\mathrm{S}$ & $\mathrm{S}$ & - & - & - \\
\hline \multicolumn{17}{|c|}{ Identified Use-Wear } \\
\hline Striations & M & $\mathrm{L}$ & - & L & L & L & S & M & M & S & - & $S$ & - & - & M & $\mathrm{s}$ \\
\hline Edge Rounding & M & - & $\mathrm{L}$ & - & L & $\mathrm{L}$ & L & M & M & M & $\mathrm{S}$ & $S$ & $\mathrm{~S}$ & $\mathrm{~S}$ & M & $\mathrm{L}$ \\
\hline Fractures & - & - & - & $\mathrm{S}$ & - & - & - & - & - & S & $\mathrm{S}$ & $S$ & $\mathrm{~S}$ & $\mathrm{~S}$ & $\mathrm{~S}$ & $\mathrm{~s}$ \\
\hline \multirow[t]{2}{*}{ Surface Polish } & - & - & - & - & $S$ & - & - & M & M & - & - & - & - & - & $\mathrm{M}$ & M \\
\hline & \multicolumn{2}{|c|}{$777-7$} & \multicolumn{2}{|c|}{$1600-3$} & \multicolumn{2}{|c|}{$1100-5$} & \multicolumn{2}{|c|}{$62600-1$} & \multicolumn{2}{|c|}{$30900-2$} & \multicolumn{2}{|c|}{$31800-1$} & 110 & & & \\
\hline $\begin{array}{l}\text { Identified } \\
\text { Residues }\end{array}$ & D & $\mathbf{v}$ & D & $\mathbf{v}$ & D & v & D & $\mathbf{v}$ & D & $\mathbf{v}$ & D & $\mathbf{v}$ & D & $\mathbf{v}$ & D & v \\
\hline Blood & - & $S$ & - & - & - & - & - & - & - & - & - & $\mathrm{M}$ & - & - & - & - \\
\hline Collagen & $\mathrm{M}$ & $S$ & M & L & M & M & $S$ & L & M & M & L & $\mathrm{L}$ & - & - & M & M \\
\hline Vivianite & - & - & - & - & - & - & - & - & - & - & - & $\mathrm{M}$ & - & - & - & - \\
\hline Feather & S & - & - & - & - & - & - & M & - & - & - & $S$ & - & - & - & - \\
\hline Hair & $S$ & - & - & $\mathrm{S}$ & - & - & - & $\mathrm{s}$ & - & - & - & $S$ & - & $\mathrm{S}$ & - & - \\
\hline Plant Tissue & - & $\mathrm{M}$ & $S$ & $\mathrm{M}$ & L & - & $\mathrm{M}$ & L & - & L & - & $\mathrm{L}$ & L & - & L & - \\
\hline Cellulose & $S$ & $S$ & - & $S$ & $S$ & - & $S$ & M & $S$ & $S$ & - & $\mathrm{M}$ & $\mathrm{S}$ & - & $\mathrm{S}$ & - \\
\hline Phytoliths & - & - & $S$ & - & - & - & - & M & - & $S$ & - & $\mathrm{M}$ & $\mathrm{s}$ & - & $\mathrm{S}$ & - \\
\hline Raphides & - & - & - & - & - & - & - & - & - & - & - & - & - & - & - & - \\
\hline Starch Granules & - & - & - & - & SG & SG & SG & MTG & ST & SG & - & ST & ST & - & - & - \\
\hline Red Resin & $\mathrm{M}$ & $\mathrm{L}$ & $\mathrm{M}$ & L & L & $\mathrm{L}$ & L & $\mathrm{M}$ & $M$ & L & $M$ & $S$ & $M$ & L & $M$ & - \\
\hline $\begin{array}{l}\text { Orange/Amber } \\
\text { Resin }\end{array}$ & $S$ & - & $S$ & $\mathrm{M}$ & $S$ & $S$ & $S$ & $\mathrm{~s}$ & $S$ & $S$ & - & - & - & $S$ & $\mathrm{~s}$ & - \\
\hline Clear/Grey Resin & $\mathrm{L}$ & $\mathrm{L}$ & $S$ & L & M & M & M & L & L & L & M & $\mathrm{L}$ & L & S & L & - \\
\hline Charred Resin & $S$ & $\mathrm{~s}$ & - & - & $\mathrm{s}$ & $\mathrm{L}$ & - & $\mathrm{s}$ & $\mathrm{S}$ & L & M & $S$ & - & - & $\mathrm{S}$ & $\mathrm{s}$ \\
\hline Charcoal & $\mathrm{M}$ & M & $S$ & L & M & M & L & M & M & M & $\mathrm{S}$ & $S$ & S & $\mathrm{S}$ & M & $\mathrm{s}$ \\
\hline Ochre & - & - & L & - & $S$ & M & - & - & $S$ & - & - & $S$ & $\mathrm{~s}$ & - & - & - \\
\hline Diatoms & - & - & - & - & - & - & - & - & - & - & - & - & - & - & $\mathrm{M}$ & $\mathrm{s}$ \\
\hline Identified Use-We & & & & & & & & & & & & & & & & \\
\hline Striations & S & $S$ & - & $\mathrm{S}$ & S & $S$ & M & M & $S$ & S & M & $\mathrm{L}$ & S & $\mathrm{S}$ & - & - \\
\hline Edge Rounding & $S$ & $S$ & $S$ & $\mathrm{~S}$ & $S$ & $S$ & $S$ & M & $S$ & $S$ & M & $S$ & L & $\mathrm{M}$ & M & $\mathrm{L}$ \\
\hline Fractures & L & $\mathrm{S}$ & - & $\mathrm{S}$ & - & - & $\mathrm{S}$ & - & $\mathrm{M}$ & $\mathrm{M}$ & $\mathrm{M}$ & $S$ & $M$ & - & $\mathrm{M}$ & - \\
\hline Surface Polish & - & $\mathrm{L}$ & - & $\mathrm{M}$ & - & - & - & - & $S$ & $S$ & $\mathrm{~S}$ & $\mathrm{M}$ & $\mathrm{S}$ & L & L & $\mathrm{s}$ \\
\hline
\end{tabular}


Table 5. Residue co-occurrence and inferred task association

\begin{tabular}{|l|l|l|}
\hline $\begin{array}{l}\text { Possible Task } \\
\text { Association }\end{array}$ & Residue Types Identified & References \\
\hline Woodworking & $\begin{array}{l}\text { Woody plant tissue, cells, cellulose, bordered pits, } \\
\text { exudate }\end{array}$ & $\begin{array}{l}\text { Hardy and Garufi } \\
(1998)\end{array}$ \\
\hline Plant Processing & Plant tissue, cellulose, phytoliths, transient starch & Briuer (1976) \\
\hline Boneworking & Bone collagen & Loy (1993) \\
\hline Charred Woodworking & Charred resin, plant fibres, cells, cellulose, charcoal & Anderson (1980) \\
\hline $\begin{array}{l}\text { Root/Tuber } \\
\text { Processing }\end{array}$ & Starch, plant tissue, cellulose, phytoliths & Fullagar et al. (1992) \\
\hline Butchery & Blood, collagen, vivianite, feather*, hair* & Loy (2000) \\
\hline $\begin{array}{l}\text { Secondary Bone- } \\
\text { working }\end{array}$ & Collagen, vivianite, feather*, hair* & Loy (2000) \\
\hline $\begin{array}{l}\text { Tertiary Bone-working } \\
\text { Bone collagen, ochre* }\end{array}$ & $\begin{array}{l}\text { Loy and Nugent } \\
\text { (2002) }\end{array}$ \\
\hline $\begin{array}{l}\text { Ceremony / } \\
\text { Decoration }\end{array}$ & Ochre, feathers, blood, hair* & Akerman et al. (2002) \\
\hline * Non-essential residue type for associated task & \\
\hline
\end{tabular}

performed with the tulas in the sample. These results concur with the ethnographic documentation cited above. Charcoal was also consistently observed in association with red and charred resin, which, as suggested above, indicates the wood may have been charred prior to being worked.

The presence of gelatinised starch and plant cellulose on the surface of an artefact suggests contact with heated or cooked starchy plants, such as corms, tubers, and rhizomes (Crowther 2001). The processing of cooked starch-rich foods using a tula has not been documented ethnographically, although a hafted tula may have served to remove food from a fire or similarly to remove the fleshy parts of the plant from its casing or skin. It is also plausible that gelatinised starch was deposited on the surface of the artefacts by touch-transfer.

Plant material was also identified in conjunction with small and moderates amounts of ochre. As suggested by Horne and Aiston (1924:109), replacement tula flakes were stored in hair, fur or plant fibre bags decorated with bands of ochre. Tindale (1965-1968:147) observed flakes and their wooden handles being decorated in ochre before they were traded, and Spencer and Gillen (1969:668) documented tula hafted woomeras being used as receptacles for holding material, such as feathers, ochre and blood for use as decoration or in ceremony. The transfer of ochre onto the tula surfaces may have been caused by any or all of these activities. Alternatively, this residue may have originated as an environmental additive, but this cannot be determined without conducting soil analyses. Blood, feather and hair, found in association with bone collagen, may also indicate butchery or fresh (secondary stage) bone-working.

As vivianite is formed in the presence of lipids such as animal fats (McGowan and Prangnell 2006) its presence with bone collagen further suggests that butchery and/or secondary stage boneworking was conducted with the tulas, tasks that are both reported in the ethnographic literature. The presence of granular bone collagen and bone pieces, occasionally with ochre, are indicative of dry (or tertiary stage) bone-working and/or decorative engraving. Although the working of dried bone with hafted tulas has not been observed ethnographically, the concentration of bone collagen on the artefact surfaces in association with relevant residues strongly suggests that boneworking at each stage was undertaken using the tulas.

\section{Use-wear}

Although the sample of tulas was small, obvious variation in the distal edge morphology was observed. This variation resonates with the ethnographies that suggested the curvature of the working edge determined the flake's primary application and, accordingly, the depth and width of the incision made. The extent of retouch exhibited on the tulas is also in direct accordance with ethnographic observation and archaeological research into tula reduction (Hiscock 1988; Kamminga 1982; Roth 1904). However, apart from the distal edge retouch evident on the slugs, 
it was difficult to conclude with any certainty which scarring was produced through manufacture or as a result of use. Similarly, the fine edge-chipping evident on the distal, lateral and proximal edges of the majority of tulas in the sample was more likely to have been produced during the final stages of reduction, as observed by Aiston (1928:127). Alternatively, and especially in view of the disturbed nature of the site, this chipping with its irregular morphology may have resulted from post-use damage (McBrearty et al. 1998; Moss 1983). The angles of the distal and dorsal platform edges on the tulas, however, are synonymous with the range of angles applied by Kamminga $(1982: 73,76)$, and thus, are suggested as suitable for efficient adzing and scraping.

The observation of particular types of scarring present on the tulas is also in accord with Kamminga's (1982) results concerning the use-wear produced from hafted adzing and scraping. The frequent occurrence of large step, feather and, to a lesser degree, hinge termination fractures along the distal edge and lateral margins on the majority of analysed artefacts indicates that these edges were used. This combination of termination fractures has been suggested by Kamminga (1982:69-78) to be a product of medium-light wood scraping and/or dense wood adzing. Moreover, artefacts displaying bending fractures may also have been employed for dense wood scraping. Edge rounding, striations, and surface polish are also indicative of use (Hayden and Kamminga 1979; Semenov 1964). Some edge and surface damage was observed in association with inferred use-related residue, such as resin and bone collagen, further suggesting the tulas' application.

\section{Edge utilisation}

By examining the distribution of use-related residues and wear patterns along the four 'working' edges, it was possible to infer particular functions performed by the tulas. Bone collagen was the most frequently occurring residue type observed along the proximal edge and red resin was the highest occurring residue type for the distal edge and lateral margins. Given that a predominance of plant material, cellulose, fibres, and mircofractures were observed along the distal edge in association with red resin, it may be inferred that this edge was primarily used for woodworking. Surface polish resembling Binneman's (1983:94) results for dried woodworking was also observed along the distal edge of most tulas. The severity of wear, the concentration of polish and the occurrence of red resin lessened towards the platform edge where the highest occurrence of bone collagen was observed.

It has been documented ethnographically that the degree of curvature of the working edge influenced the flake's application to a particular stage of woodworking (Aiston 1928:125; McCarthy 1967:29; Spencer and Gillen 1969:637-9). Furthermore, Kamminga (1982:77) reported that a curved working edge is necessary to lessen the impact of dynamically loaded functions such as hafted adzing. These observations support the suggestion that woodworking activities conducted with the tulas in the sample were confined to the curved perimeter edges of the distal edge and upper lateral margins. The presence along the platform edge of bone collagen with fine striations and compression ring fractures, which are generally associated with scraping (Kamminga 1982:70), suggests that bone-working activities may not require a curved edge. Such evidence also implies predetermined tula manufacture.

\section{Evidence of hafting}

As recorded in the ethnographic literature, tulas functioned primarily as hafted composite tools. Therefore, the presence of resinous material identified on all of the tulas may not only be associated with woodworking, but also hafting. Inferences concerning hafting, including the orientation of the flake in the haft and multiple hafting arrangements, were determined by analysing the nature, location and frequency of the types of resins observed. It is unlikely that all five resins types identified represent separate hafting mediums. The resin types more likely attributable to woodworking are red, charred and purple resin, and those suggested here as hafting mediums, are clear/grey and orange/amber resin.

According to ethnographic observations, both the distal and platform edges were primary working edges for adzing and scraping, having both acute edge angles adjacent to the convex 
ventral surface. This patterning is particularly evident on the slugs, supporting the idea that continued use of the flake necessitated the utilisation of the proximal end. Furthermore, both red and charred resin occur predominantly in linear smears perpendicular or sub-perpendicular to these edges and occasionally adjacent to or caught within edge microfractures. Linear smears, as opposed to a scattered distribution of residue, are generally indicative of the artefact's movement along the work surface and similarly through the residue. Accordingly, the specific resin patterning and location on the tula's surface may be the products of adzing or scraping red resinous wood. The presence of charred resin may indicate prior charring of the wood to facilitate smoothing and shaping, as recorded in the ethnographic literature (Thomson 1975:98; Tindale 1972). Tree species common to Camooweal and the surrounding area that produce a red resinous exudate are desert or western bloodwood (Corymbia terminalis), red bloodwood (Eucalyptus gummifera), and beefwood (Grevillea striata).

Clear/grey resin was observed in similar quantities to red resin, although differences in appearance and location on the tulas' surfaces suggest this resin is more likely to be the remnants of hafting rather than woodworking. Unlike the red and charred resins, clear/grey resin did not display evidence of direction of application in the form of striations or linear smearing but generally occurs in dense, cracked patches and scattered deposits across the artefact surface. This pattern suggests the resin was applied to the surface of the artefact directly such as a hafting medium rather than resulting from artefact use. Comparison with samples of spinifex resin (Triodia pungens) obtained from Camooweal revealed similarities in appearance, suggesting clear/grey resin may have originated from this species. In comparison, orange/amber resin was observed in similar deposits and patterning along the lateral margins and percussion bulb but in much smaller quantities and less frequently across the sample. Orange/amber resin is similar in appearance to grass tree resin (Xanthorrhoea sp.), which has also been ethnographically observed as a hafting medium (Gott and Conran 1991). Although its presence or use has not been documented at Camooweal, grass tree resin may have been traded into the area.

The distribution of clear/grey resin across the tula surfaces, in association with use-related residues and wear patterns on all perimeter edges, strongly suggests that these artefacts were hafted in more than one orientation to the handle. This distribution concurs with the ethnographic literature where it was noted that a combination of hafting arrangements were employed at different stages of tula manufacture, (re)sharpening, and use (Tindale 1965-1968:154).

The resin patterning on $999.428,66800-3,62600-1,60400-2$ and $9800-4$ indicates that these tulas were hafted at some point to utilise the proximal end, given the concentration of clear/ grey resin along the lower mid section and distal edge. Artefacts 1100-4a and 1600-3 display evidence of a distal hafting arrangement, and artefacts 22300-4, 34700-3, 1100-4b were once hafted to utilise the lateral margins. The remaining tulas in the sample display either a fairly even distribution of clear/grey resin across the surface, or insufficient quantities to infer the orientation of the flake in the haft. An increase in frequency of hafting resin toward a particular edge may indicate the most recent hafting arrangement prior to discard, as subsequent use of a rehafted flake may remove some, if not the majority, of resin from prior hafts. Prior hafting is indicated by the presence of use-related residues and wear patterns on more than one edge in association with the proposed hafting resin. A scattered distribution of resin across the artefact surface in association with use-related residues and wear patterns along all edges suggests that a combination of hafting arrangements were employed. In the absence of hafting resin, it may be inferred that artefacts were either used unhafted or that exposure to post-depositional processes removed this evidence.

\section{Inferred functions and task associations}

Integration and comparison of results of the macroscopic, low-magnification and highmagnification examinations of the tulas enabled their possible hafting arrangements, functions and task associations to be determined (see Table 6). These results suggest that all 16 tulas were employed not only for woodworking but also for butchery and secondary and tertiary bone-working. Possible woodworking tasks include the manufacture of water carriers, digging bowls and other 
Table 6. Inferred hafting arrangement, functions and task associations

\begin{tabular}{|c|c|c|c|c|c|c|c|c|c|c|c|}
\hline $\begin{array}{l}\text { Artefact } \\
\text { number }\end{array}$ & $\begin{array}{c}\text { Inferred } \\
\text { hafting } \\
\text { arrange. }\end{array}$ & $\begin{array}{l}\text { Inferred } \\
\text { function }\end{array}$ & $\begin{array}{l}\text { Wood- } \\
\text { work. }\end{array}$ & $\begin{array}{c}\text { Plant } \\
\text { process. }\end{array}$ & $\begin{array}{l}\text { Bone- } \\
\text { work. }\end{array}$ & $\begin{array}{l}\text { Charred } \\
\text { wood- } \\
\text { work. }\end{array}$ & $\begin{array}{c}\text { Tuber } \\
\text { process. }\end{array}$ & Butchery & $\begin{array}{c}\text { Secondary } \\
\text { bone- } \\
\text { working }\end{array}$ & $\begin{array}{c}\text { Tertiary } \\
\text { bone- } \\
\text { working }\end{array}$ & $\begin{array}{l}\text { Ceremony/ } \\
\text { decoration }\end{array}$ \\
\hline $\begin{array}{l}20100- \\
7 a\end{array}$ & Inconclusive & Scraping & Yes & Yes & Yes & Yes & Yes & & & Yes & Yes \\
\hline $22300-4$ & Lateral & $\begin{array}{l}\text { Adze / } \\
\text { Scrape }\end{array}$ & Yes & Yes & Yes & Yes & & Yes & Yes & Yes & \\
\hline 999.428 & Proximal & Scraping & Yes & Yes & Yes & Yes & & & Yes & Yes & Yes \\
\hline $34700-3$ & Lateral & $\begin{array}{l}\text { Adze / } \\
\text { Scrape }\end{array}$ & Yes & Yes & Yes & Yes & Yes & Yes & Yes & & \\
\hline $66800-3$ & Proximal & $\begin{array}{l}\text { Adze / } \\
\text { Scrape }\end{array}$ & Yes & Yes & Yes & Yes & Yes & & Yes & Yes & \\
\hline $60400-2$ & Proximal & $\begin{array}{l}\text { Adze / } \\
\text { Scrape }\end{array}$ & Yes & & Yes & Yes & & Possible & & Yes & \\
\hline $\begin{array}{l}20100- \\
7 \mathrm{~b}\end{array}$ & Inconclusive & Scraping & Yes & Yes & Yes & & Yes & Possible & Possible & Yes & \\
\hline $1100-4 a$ & Distal & Scraping & Yes & & Yes & Yes & & Possible & & & Yes \\
\hline $777-7$ & Prox /Dist & $\begin{array}{l}\text { Adze / } \\
\text { Scrape }\end{array}$ & Yes & & Yes & Yes & & Possible & & & Yes \\
\hline $1600-3$ & Distal & Scraping & Yes & Yes & Yes & Yes & & & & Yes & \\
\hline $1100-5$ & Inconclusive & Scraping & Yes & & Yes & Yes & & & & Yes & \\
\hline $62600-1$ & Proximal & Scraping & Yes & Yes & Yes & Yes & Yes & & & Yes & \\
\hline $30900-2$ & Lat / Prox & $\begin{array}{l}\text { Adze / } \\
\text { Scrape }\end{array}$ & Yes & & Yes & Yes & & & & Yes & \\
\hline $31800-1$ & Prox / Dist & $\begin{array}{l}\text { Adze / } \\
\text { Scrape }\end{array}$ & Yes & Yes & Yes & Yes & & Yes & Yes & Yes & \\
\hline $1100-4 b$ & Lateral & Scraping & Yes & & Possible & & & & & & \\
\hline $9800-4$ & Proximal & Scraping & Yes & & Yes & Yes & & & & Yes & \\
\hline
\end{tabular}

items from local tree species such as Corymbia terminalis, Eucalyptus gummifera, and Grevillea striata, The tulas were also hafted to the handle in several orientations, possibly with spinifex (Triodia $\mathrm{sp.)}$ resin or grass tree resin (Xanthorrhoea sp.). Alternating hafting arrangements allowed for the use of all 'working' edges, thereby increasing the inherent functionality, adaptability and utilitarian 'use-life' of the tula.

\section{Why analyse surface collected artefacts?}

Some of the most abundant and diverse archaeological information in Australia lies on the surface (Fanning and Holdaway 2001:668; Hiscock and Hughes 1983:87). Nevertheless, a large proportion of this information remains hidden beneath the labels of 'disturbed', 'complex' and 'exposed'. Traditionally, these views have fuelled the perception that surface assemblages lack the integrity of subsurface archaeological deposits. Consequently, their full potential as an important component of the archaeological record has not always been realised (Fanning and Holdaway 2001:668; Lewarch and O'Brien 1981:297). Much of the rationale behind these statements was based on the assumption that subsurface materials are 'fossilised' records of the past, and that post-depositional disturbance processes acting upon surface material are apparently unique to contemporary ground surfaces and do not affect subsurface deposits (Fanning and Holdaway 2001; Lewarch and O'Brien 1981:312). However, it has been more than two decades since Dunnell and Dancy (1983) made the observation that all archaeological deposits now buried were once 'exposed' on the surface, albeit for a lesser period of time. In addition to factors that affect artefact use, curation, reuse and discard, post-depositional natural and cultural processes continually modify the content, condition and patterning of archaeological deposits (Lewarch and O'Brien 1981:312; Schiffer 1987). As a result few artefacts, surface or subsurface, are in situ in the conventional sense of the term (Foley 1981:170). Based on the consistent indications of tool use on both the labelled excavated and surface collected artefacts presented above, we argue traces of use are as likely to survive post-depositional disturbance processes in either context.

\section{CONCLUSION}

The results of the initial residue and use-wear analyses of 23 stone artefacts from the Georgina River Bridge site, Camooweal, suggest similar uses for certain tool types, whether they had been 
excavated or surface collected. The subsequent detailed examination of 16 surface collected tulas identified traces of use and a wide variety of residues indicative of multiple hafting episodes and specific tasks performed, included woodworking, plant processing, bone-working and butchery. Examination of these results in light of ethnographic documentation and previous archaeological research suggests the manufacture of the tula was predetermined to achieve certain tasks, and that these artefacts may have been used for several tasks before being discarded. The consistent indications of tool use found in both studies strongly supports the application of use-wear and residue analyses as suitable methods for examining stone artefacts recovered from complex and disturbed surface sites.

\section{ACKNOWLEDGEMENTS}

We are sincerely grateful for the inspiration and guidance always generously provided by Tom Loy. Thank you to Colin Saltmere and the Indjilandji People from the Camooweal area for access to the artefacts and permission to publish the results of the preliminary research. In addition, we acknowledge Ann Wallin for permission to cite from the Archaeo Cultural Heritage Services' report 'Georgina River Bridge Cultural Heritage Project'. We also thank members of Tom's Group for encouragement and support, and especially Dr Gail Robertson for comments on drafts of this paper. Funding for the research was provided by the School of Social Science at the University of Queensland, and Archaeo Cultural Heritage Services.

\section{REFERENCES}

Aiston, G. 1928. Chipped stone tools of the Aboriginal tribes east and north-east of Lake Eyre, South Australia. Papers and Proceedings of the Royal Society of Tasmania 123-131.

Aiston, G. 1929. Method of mounting stone tools on Koondi: tribes east and north-east of Lake Eyre. Papers and Proceedings of the Royal Society of Tasmania 44-46.

Akerman, K., R. Fullagar and A. van Gijn 2002. Weapons and wunan: production, function and exchange of Kimberley points. Australian Aboriginal Studies 1:13-42.

Anderson, P. 1980. A testimony of prehistoric tasks: diagnostic residues on stone tool edges. World Archaeology 12:181-194

Archaeo Cultural Heritage Services and Dugalunji Aboriginal Corporation. 2002. Georgina River Bridge Cultural Heritage Project. Report to the Department of Main Roads, Queensland.

Binneman, J. 1983. Microscopic examination of a hafted tool. South African Archaeological Bulletin 38:93-95.

Briuer, F. 1976. New clues to stone tool function: plant and animal residues. American Antiquity 41:478-484.

Campbell, T. and R. Edwards 1966. Stone implements. In B.C. Cotton (ed.) Aboriginal Man in south and central Australia, Part 1, pp. 159-220. Adelaide: South Australian Government Printer.

Canti, M. 1998. The micromorphological identification of faecal spherulites from archaeological and modern materials. Journal of Archaeological Science 25:435-444.

Crowther, A. 2001. Pots, Plants and Pacific Prehistory. Unpublished B.A. Honours thesis. St Lucia: School of Social Science, The University of Queensland.

Davidson, D.S. 1935. Archaeological problems of northern Australia. The Journal of the Royal Anthropological Institute of Great Britain and Ireland 65:145-186. 
Davis, E. 1975. The "exposed archaeology" of China Lake, California. American Antiquity 40:3953.

Dunnell, R.C. and W. Dancey 1983. The siteless survey: a regional scale data collection strategy. Advances in Archaeological Method and Theory 6:267-287.

Ebert, J. 1992. Distributional Archaeology. University of New Mexico Press, Albuquerque.

Etheridge, R. 1891. Notes of Australian Aboriginal stone weapons and implements. Proceedings of the Linnaean Society of New South Wales 6:31-43.

Fanning, P. and S. Holdaway 2001. Stone artefact scatters in western NSW, Australia: geomorphic controls on artefact size and distribution. Geoarchaeology 16:667-686.

Foley, R. 1981 . Off-site archaeology: an alternative approach for the short-sited. In G. Hodder, G. Isaac and N. Hammond (eds) Patterns of the Past, Studies in Honour of David Clarke, pp. 157-183. Cambridge University Press, Cambridge.

Fullagar, R., B. Meehan and R. Jones 1992. Residue analysis of ethnographic plant-working and other tools from Northern Australia. Préhistoire de l'Agriculture : Nouvelle Approches Expérimentales et Ethnographiques. Monographie du CRA No. 6, pp.40-53.

Gott, B. and J. Conran 1991. Victorian Koorie Plants: Some Plants Used by Victorian Koories for Food, Fibre, Medicines and Implements. Hamilton: Yangennanock Womens Group.

Gould, R. 1978. The anthropology of human residue. American Anthropologist 80:815-835.

Gould, R., K. Koster and A. Sontz 1971. The lithic assemblage of the Western Desert Aborigines of Australia. American Antiquity 36:149-169.

Greenfield, H.J. 2000. Integrating surface and subsurface reconnaissance data in the study of stratigraphically complex sites: Blagotin, Serbia. Geoarchaeology 15:167-201.

Hardy, B.L. and G.T. Garufi 1998. Identification of woodworking on stone tools through residue and use-wear analysis: experimental results. Journal of Archaeological Science 25:177184.

Hayden, B. and J. Kamminga 1979. An introduction to use-wear: the first CLUW. In B. Hayden (ed.) Lithic Use-wear Analysis, pp. 1-13. New York: Academic Press.

Hiscock, P. 1988. A cache of tulas from the Boulia District, Western Queensland. Archaeology in Oceania 23:60-70.

Hiscock, P. 1994. Technological responses to risk in Holocene Australia. Journal of World Prehistory 8:267-292.

Hiscock, P. and P.J. Hughes 1983. One method of recording scatters of stone artefacts during site surveys. Australian Archaeology 17:87-98.

Horne, G. and G. Aiston 1924. Savage Life in Central Australia. London: Macmillan.

Kamminga, J. 1982. Over the edge: Functional Analysis of Australian Stone Tools. Occasional Papers in Anthropology No 12. St. Lucia: The University of Queensland Printery.

Lewarch, D. and M. O'Brien 1981. The expanding role of surface assemblages in archaeological research. Advances in Archaeological Method and Theory 4:297-342.

Loy, T.H. 1983. Prehistoric blood residues: detection on tool surfaces and identification of species of origin. Science 220:1269-1271. 
Loy, T.H. 1993. The artefact as a site: an example of the biomolecular analysis of organic residues on prehistoric tools. World Archaeology 25(No 1):44-63.

Loy, T.H. 2000. Analysis of six pottery sherds from the west coast of Guam. Report to Micronesian Archaeological Research Services, Guam.

Loy, T.H. and S.J. Nugent 2002. Residue analysis of a portion of the Georgina River Bridge collections, Camooweal, Queensland. Report to Archaeo Cultural Heritage Services Pty. Ltd. Brisbane.

McBrearty, S., L. Bishop, T. Plummer, R. Dewar and N. Conard 1998. Tools underfoot: human trampling as an agent of lithic artefact edge modification. American Antiquity 63:108129.

McCarthy, F.D. 1967. Australian Aboriginal Stone Implements. Sydney: Museum Trust.

McGowan, G. and J. Prangnell. 2006. The significance of vivianite in archaeological settings. Geoarchaeology 21:93-111.

McNiven, I. 1993. Tula adzes and bifacial points on the east coast of Australia. Australian Archaeology 36:22-31.

Mitchell, S.R. 1949. Stone-age Craftsmen: Stone Tools and Camping Places of the Australian Aborigines. Melbourne: Tait Book Co. Pty. Ltd.

Moore, M.W. 2003. Flexibility of stone tool manufacturing methods on the Georgina River, Camooweal, Queensland. Archaeology in Oceania 38:23-36.

Moss, E.H. 1983. Some comments on edge damage as a factor in functional analysis of stone tools. Journal of Archaeological Science 10:231-242.

Orr, D.M. and W.E. Holmes 1990. Mitchell grasslands. In G.N. Harrington, A.D. Wilson and M.D. Young (eds) Management of Australia's Rangelands, pp. 241-254. Melbourne: Division of Wildlife and Ecology, CSIRO.

Raven, P.H., R.F. Evert and S.E. Eichorn 1999. Biology of Plants. New York: W. H. Freeman and Company.

Rossignol, J. and L. Wandsnider 1992. Space, Time and Archaeological Landscapes. New York: Plenum Press.

Roth, W.E. 1897. Ethnological Studies Among the North-west-central Queensland Aborigines. Brisbane: Government Printer.

Roth, W.E. 1904. Domestic Implements, Arts and Manufactures. North Queensland Ethnography: Bulletin No.7. Brisbane: George Arthur Vaughan, Government Printer.

Schiffer, M.B. 1987. Formation Processes of the Archaeological Record. Albuquerque: University of New Mexico Press.

Semenov, S. 1964. Prehistoric Technology. Cory, Adams and Mackay, London.

Spencer, B. and F.J. Gillen 1969. Northern Tribes of Central Australia. Oosterhout:Anthropological Publications.

Sullivan, A.P. 1998. Surface Archaeology. Albuquerque: University of New Mexico Press.

Thomson, D.F. 1964. Some wood and stone implements of the Bindibu tribe of central Western Australia. Proceedings of the Prehistoric Society 30:400-422. 
Thomson, D.F. 1975. Bindibu Country. Melbourne: Thomas Nelson.

Tindale, N.B. 1965-1968. Stone implement making among the Nakako, Ngadadjara and Pitjandjara of the Great Western Desert. Records of the South Australian Museum Vol 15:131-164.

Tindale, N.B. 1972. The Pitjandjara. In M.G. Bicchieri (ed.) Hunters and Gatherers Today, pp. 217-268. New York: Holt Rinehart and Winston Inc.

Wilkinson, T.J. 2001. Surface collection techniques in field archaeology. In D.R. Brothwell and A.M. Pollard (eds) Handbook of Archaeological Sciences, Chichester: Wiley and Sons Ltd. 